\title{
Quantum Logic in Dagger Kernel Categories
}

\author{
Chris Heunen • Bart Jacobs
}

Received: 13 June 2009 / Accepted: 26 January 2010 / Published online: 17 February 2010

(C) The Author(s) 2010. This article is published with open access at Springerlink.com

\begin{abstract}
This paper investigates quantum logic from the perspective of categorical logic, and starts from minimal assumptions, namely the existence of involutions/daggers and kernels. The resulting structures turn out to (1) encompass many examples of interest, such as categories of relations, partial injections, Hilbert spaces (also modulo phase), and Boolean algebras, and (2) have interesting categorical/logical/order-theoretic properties, in terms of kernel fibrations, such as existence of pullbacks, factorisation, orthomodularity, atomicity and completeness. For instance, the Sasaki hook and and-then connectives are obtained, as adjoints, via the existential-pullback adjunction between fibres.
\end{abstract}

Keywords Quantum logic·Dagger kernel category • Orthomodular lattice • Categorical logic

\section{Introduction}

Dagger categories $\mathbf{D}$ come equipped with a special functor $\dagger: \mathbf{D}^{\text {op }} \rightarrow \mathbf{D}$ with $X^{\dagger}=X$ on objects and $f^{\dagger \dagger}=f$ on morphisms. A simple example is the category Rel of sets and relations, where $\dagger$ is reversal of relations. A less trivial example is the category Hilb of Hilbert spaces and continuous linear transformations, where $\dagger$ is induced

Chris Heunen was supported by the Netherlands Organisation for Scientific Research (NWO).

C. Heunen $(\bowtie)$

Oxford University Computing Laboratory, Oxford, UK

e-mail: chris.heunen@comlab.ox.ac.uk

B. Jacobs

Institute for Computing and Information Sciences (iCIS), Radboud University Nijmegen,

Nijmegen, The Netherlands 
by the inner product. The use of daggers, mostly with additional assumptions, dates back to $[31,35]$. Daggers are currently of interest in the context of quantum computation $[1,7,40]$. The dagger abstractly captures the reversal of a computation.

Mostly, dagger categories are used with fairly strong additional assumptions, like compact closure in [1]. Here we wish to follow a different approach and start from minimal assumptions. This paper is a first step to understand quantum logic, from the perspective of categorical logic (see e.g. [21, 28, 32, 41]). It grew from the work of one of the authors [18]. Although that paper enjoys a satisfactory relation to traditional quantum logic [19], this one generalises it, by taking the notion of dagger category as starting point, and adding kernels, to be used as predicates. The interesting thing is that in the presence of a dagger $\dagger$ much else can be derived. As usual, it is quite subtle what precisely to take as primitive. A referee identified the reference [9] as an earlier precursor to this work. It contains some crucial ingredients, like orthomodular posets of dagger kernels, but without the general perspective given by categorical logic.

Upon this structure of "dagger kernel categories" the paper constructs pullbacks of kernels and factorisation (both similar to [14]). It thus turns out that the kernels form a "bifibration" (both a fibration and an opfibration, see [21]). This structure can be used as a basis for categorical logic, which captures substitution in predicates by reindexing (pullback) $f^{-1}$ and existential quantification by op-reindexing $\exists_{f}$, in such a way that $\exists_{f} \dashv f^{-1}$. From time to time we use fibred terminology in this paper, but familiarity with this fibred setting is not essential. We find that the posets of kernels (fibres) are automatically orthomodular lattices [26], and that the Sasaki hook and and-then connectives appear naturally from the existential-pullback adjunction. Additionally, a notion of Booleanness is identified for these dagger kernel categories. It gives rise to a generic construction that generalises how the category of partial injections can be obtained from the category of relations.

Apart from this general theory, the paper brings several important examples within the same setting - of dagger kernel categories. Examples are the categories Rel and PInj of relations and partial injections. Additionally, the category Hilb is an example —and, interestingly —also the category PHilb of Hilbert spaces modulo phase. The latter category provides the framework in which physicists typically work [6]. It has much weaker categorical structure than Hilb. We also present a construction to turn an arbitrary Boolean algebra into a dagger kernel category.

The authors are acutely aware of the fact that several of the example categories have much richer structure, involving for instance a tensor sum $\oplus$ and a tensor product $\otimes$ with associated scalars and traced monoidal structure. This paper deliberately concentrates solely on (the logic of) kernels. There are interesting differences between our main examples: for instance, Rel and PInj are Boolean, but Hilb is not; in PInj and Hilb "zero-epis" are epis, but not in Rel; Rel and Hilb have biproducts, but PInj does not.

The paper is organised as follows. After introducing the notion of dagger kernel category in Section 2, the main examples are described in Section 3. Factorisation and (co)images occur in Sections 4 and 5. Section 6 introduces the Sasaki hook and and-then connectives via adjunctions, and investigates Booleanness. Finally, Sections 7 and 8 investigate some order-theoretic aspects of homsets and of kernel posets (atomicity and completeness).

A follow-up paper [22] introduces a new category OMLatGal of orthomodular lattices with Galois connections between them, shows that it is a dagger kernel 
category, and that every dagger kernel category D maps into it via the kernel functor KSub: D $\rightarrow$ OMLatGal, preserving the dagger kernel structure. This gives a wider context.

\section{Daggers and Kernels}

Let us start by introducing the object of study of this paper.

Definition 1 A dagger kernel category consists of:

1. a dagger category $\mathbf{D}$, with dagger $\uparrow: \mathbf{D}^{\text {op }} \rightarrow \mathbf{D}$;

2. a zero object 0 in $\mathbf{D}$;

3. kernels $\operatorname{ker}(f)$ of arbitrary maps $f$ in $\mathbf{D}$, which are dagger monos.

A morphism of dagger kernel categories is a functor $F$ preserving the relevant structure:

1. $F\left(f^{\dagger}\right)=F(f)^{\dagger}$;

2. $F(0)$ is again a zero object;

3. $F(k)$ is a kernel of $F(f)$ if $k$ is a kernel of $f$.

Dagger kernel categories and their morphisms form a category DagKerCat.

Definition 2 A dagger kernel category is called Boolean if $m \wedge n=0$ implies $m^{\dagger} \circ$ $n=0$, for all kernels $m, n$.

The name Boolean will be explained in Theorem 1. We shall later rephrase the Booleanness condition as: kernels are disjoint if and only if they are orthogonal, see Lemma 1.

The dagger $\dagger$ satisfies $X^{\dagger}=X$ on objects and $f^{\dagger \dagger}=f$ on morphisms. It comes with a number of definitions. A map $f$ in $\mathbf{D}$ is called a dagger mono(morphism) if $f^{\dagger} \circ f=\mathrm{id}$ and a dagger epi(morphism) if $f \circ f^{\dagger}=\mathrm{id}$. Hence $f$ is a dagger mono if and only if $f^{\dagger}$ is a dagger epi. A map $f$ is a dagger iso(morphism) when it is both dagger monic and dagger epic; in that case $f^{-1}=f^{\dagger}$ and $f$ is sometimes called unitary (in analogy with Hilbert spaces). An endomorphism $p: X \rightarrow X$ is called selfadjoint if $p^{\dagger}=p$.

The zero object $0 \in \mathbf{D}$ is by definition both initial and final. Actually, in the presence of $\dagger$, initiality implies finality, and vice-versa. For an arbitrary object $X \in \mathbf{D}$, the unique map $X \rightarrow 0$ is then a dagger epi and the unique map $0 \rightarrow X$ is a dagger mono. The "zero" map $0=0_{X, Y}=(X \rightarrow 0 \rightarrow Y)$ satisfies $\left(0_{X, Y}\right)^{\dagger}=0_{Y, X}$. Notice that $f \circ 0=0=0 \circ g$. Usually there is no confusion between 0 as zero object and 0 as zero map. Two maps $f: X \rightarrow Z$ and $g: Y \rightarrow Z$ with common codomain are called orthogonal, written as $f \perp g$, if $g^{\dagger} \circ f=0$-or, equivalently, $f^{\dagger} \circ g=0$.

Let us recall that a kernel of a morphism $f: X \rightarrow Y$ is a universal morphism $k: \operatorname{ker}(f) \rightarrow X$ with $f \circ k=0$. Universality means that for an arbitrary $g: Z \rightarrow X$ 
with $f \circ g=0$ there is a unique map $g^{\prime}: Z \rightarrow \operatorname{ker}(f)$ with $k \circ g^{\prime}=g$. Kernels are automatically (ordinary) monos. Just like we write 0 both for a zero object and for a zero map, we often write $\operatorname{ker}(f)$ to denote either a kernel map, or the domain object of a kernel map.

Definition 1 requires that kernels are dagger monos. This requirement involves a subtlety: kernels are closed under arbitrary isomorphisms but dagger monos are only closed under dagger isomorphisms. Hence we should be more careful in this requirement. What we really mean in Definition 1 is that for each map $f$, among all its isomorphic kernel maps, there is at least one dagger mono. We typically choose this dagger mono as representant $\operatorname{ker}(f)$ of the equivalence class of kernel maps.

We shall write $\mathrm{KSub}(X)$ for the poset of (equivalence classes) of kernels with codomain $X$. The order $(M \longmapsto X) \leq(N \longmapsto X)$ in $\operatorname{KSub}(X)$ is given by the presence of a (necessarily unique) map $M \rightarrow N$ making the obvious triangle commute. Intersections in posets like $\mathrm{KSub}(X)$, if they exist, are given by pullbacks, as in:

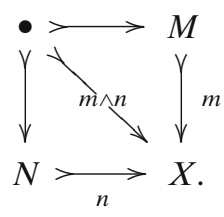

In presence of the dagger $\dagger$, cokernels come for free: one can define a cokernel coker $(f)$ as $\operatorname{ker}\left(f^{\dagger}\right)^{\dagger}$. Notice that we now write $\operatorname{ker}(f)$ and coker $(f)$ as morphisms. This cokernel coker $(f)$ is a dagger epi. Finally, we define $m^{\perp}=\operatorname{ker}\left(m^{\dagger}\right)$, which we often write as $m^{\perp}: M^{\perp} \longmapsto X$ if $m: M \longmapsto X$. This notation is especially used when $m$ is a mono. In diagrams we typically write a kernel as $\longmapsto$ and a cokernel as $\longrightarrow$.

The following Lemma gives some basic observations.

Lemma 1 In a dagger kernel category,

1. $\operatorname{ker}(X \stackrel{0}{\rightarrow} Y)=(X \stackrel{\text { id }}{\rightarrow} X)$ and $\operatorname{ker}(X \stackrel{\text { id }}{\rightarrow} X)=(0 \stackrel{0}{\rightarrow} X)$; these yield greatest and least elements $1,0 \in \mathrm{KSub}(X)$, respectively;

2. $\operatorname{ker}(\operatorname{ker}(f))=0$;

3. $\operatorname{ker}(\operatorname{coker}(\operatorname{ker}(f)))=\operatorname{ker}(f)$, as subobjects;

4. $m^{\perp \perp}=m$ if $m$ is a kernel;

5. A map $f$ factors through $g^{\perp}$ iff $f \perp g$ iff $g \perp f$ iff $g$ factors through $f_{\simeq}^{\perp}$; in particular $m \leq n^{\perp}$ iff $n \leq m^{\perp}$, for monos $m, n$; hence $(-)^{\perp}: \operatorname{KSub}(X) \stackrel{\cong}{\longrightarrow}$ $\operatorname{KSub}(X)^{\text {op }}$;

6. if $m \leq n$, for monos $m, n$, say via $m=n \circ \varphi$, then:

a. if $m, n$ are dagger monic, then so is $\varphi$;

b. if $m$ is a kernel, then so is $\varphi$.

7. Booleanness amounts to $m \wedge n=0 \Leftrightarrow m \perp n$, i.e. disjointness is orthogonality, for kernels. 
Proof We skip the first two points because they are obvious and start with the third one. Consider the following diagram for an arbitrary $f: X \rightarrow Y$ :

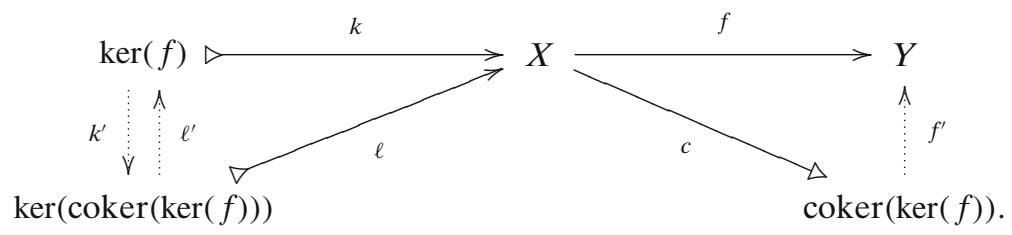

By construction $f \circ k=0$ and $c \circ k=0$. Hence there are $f^{\prime}$ and $k^{\prime}$ as indicated. Since $f \circ \ell=f^{\prime} \circ c \circ \ell=f^{\prime} \circ 0=0$ one gets $\ell^{\prime}$. Hence the kernels $\ell$ and $k$ represent the same subobject.

For the fourth point, notice that if $m=\operatorname{ker}(f)$, then

$$
m^{\perp \perp}=\operatorname{ker}\left(\operatorname{ker}\left(m^{\dagger}\right)^{\dagger}\right)=\operatorname{ker}(\operatorname{coker}(\operatorname{ker}(f)))=\operatorname{ker}(f)=m .
$$

Next,

$$
\begin{aligned}
f \text { factors through } g^{\perp} & \Longleftrightarrow g^{\dagger} \circ f=0 \\
& \Longleftrightarrow f^{\dagger} \circ g=0 \Longleftrightarrow g \text { factors through } f^{\perp} .
\end{aligned}
$$

If, in the sixth point, $m=n \circ \varphi$ and $m, n$ are dagger monos, then $\varphi^{\dagger} \circ \varphi=\left(n^{\dagger} \circ\right.$ $m)^{\dagger} \circ \varphi=m^{\dagger} \circ n \circ \varphi=m^{\dagger} \circ m=$ id. And if $m=\operatorname{ker}(f)$, then $\varphi=\operatorname{ker}(f \circ n)$, since: (1) $f \circ n \circ \varphi=f \circ m=0$, and (2) if $f \circ n \circ g=0$, then there is a $\psi$ with $m \circ \psi=$ $n \circ g$, and this gives a unique $\psi$ with $\varphi \circ \psi=g$, where uniqueness of this $\psi$ comes from $\varphi$ being monic.

Finally, Booleanness means that $m \wedge n=0$ implies $m^{\dagger} \circ n=0$, which is equivalent to $n^{\dagger} \circ m=0$, which is $m \perp n$ by definition. The reverse implication is easy, using that the meet $\wedge$ of monos is given by pullback: if $m \circ f=n \circ g$, then $f=m^{\dagger} \circ m \circ$ $f=m^{\dagger} \circ n \circ g=0 \circ g=0$. Similarly, $g=0$. Hence the zero object 0 is the pullback $m \wedge n$ of $m, n$.

Certain constructions from the theory of Abelian categories [14] also work in the current setting. This applies to the pullback construction in the next result, but also, to a certain extent, to the factorisation of Section 4.

Lemma 2 Pullbacks of kernels exist, and are kernels again. Explicitly, given a kernel $n$ and map $f$ one obtains a pullback:

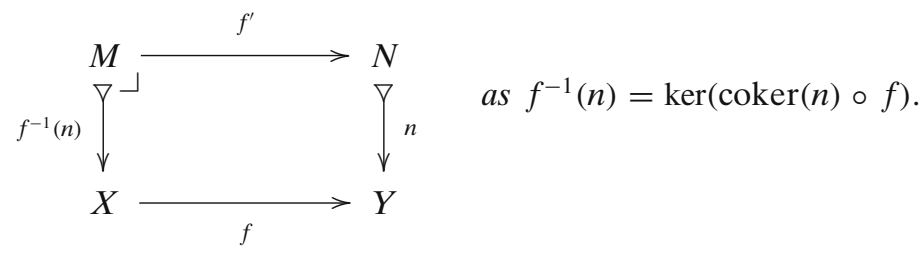

If $f$ is a dagger epi, so is $f^{\prime}$.

By duality there are of course similar results about pushouts of cokernels. 
Proof For convenience write $m$ for the dagger kernel $f^{-1}(n)=\operatorname{ker}(\operatorname{coker}(n) \circ f)$. By construction, $\operatorname{coker}(n) \circ f \circ m=0$, so that $f \circ m$ factors through $\operatorname{ker}(\operatorname{coker}(n))=n$, say via $f^{\prime}: M \rightarrow N$ with $n \circ f^{\prime}=f \circ m$, as in the diagram. This yields a pullback: if $a: Z \rightarrow X$ and $b: Z \rightarrow N$ satisfy $f \circ a=n \circ b$, then $\operatorname{coker}(n) \circ f \circ a=\operatorname{coker}(n) \circ$ $n \circ b=0 \circ b=0$, so that there is a unique map $c: Z \rightarrow M$ with $m \circ c=a$. Then $f^{\prime} \circ c=b$ because $n$ is monic.

In case $f$ is dagger epic, $f \circ f^{\dagger} \circ n=n$. Hence there is a morphism $f^{\prime \prime}$ making following diagram commute, as the right square is a pullback:

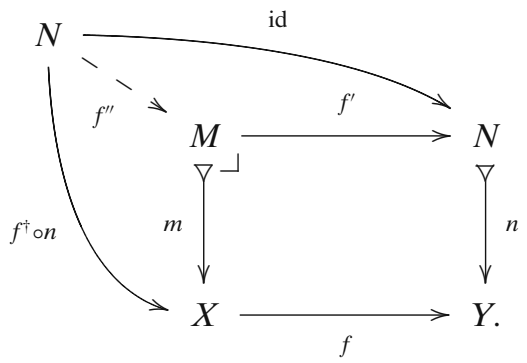

Then $f^{\prime \prime}=m^{\dagger} \circ m \circ f^{\prime \prime}=m^{\dagger} \circ f^{\dagger} \circ n=(f \circ m)^{\dagger} \circ n=\left(n \circ f^{\prime}\right)^{\dagger} \circ n=f^{\prime \dagger} \circ n^{\dagger} \circ n=$ $f^{\prime \dagger}$. Hence $f^{\prime}$ is dagger epic, too.

Corollary 1 Given these pullbacks of kernels we observe the following.

1. The mapping $X \mapsto \mathrm{KSub}(X)$ yields an indexed category $\mathbf{D}^{\mathrm{op}} \rightarrow$ PoSets, using that each map $f: X \rightarrow Y$ in D yields a pullback (or substitution) functor $f^{-1}: \operatorname{KSub}(Y) \rightarrow \operatorname{KSub}(X)$. By the "pullback lemma”, see e.g. [2, Lemma 5.10] or [29, III, 4, Exc. 8], such functors $f^{-1}$ preserve the order on kernels, and also preserve all meets (given by pullbacks). This (posetal) indexed category KSub: $\mathbf{D}^{\text {op }} \rightarrow$ PoSets forms a setting in which one can develop categorical logic for dagger categories, see Section 2.1.

2. The following diagram is a pullback,

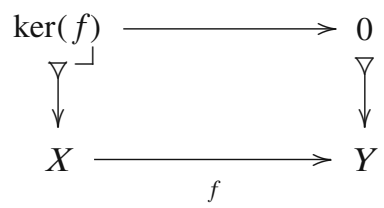

showing that, logically speaking, falsum-i.e. the bottom element $0 \in \mathrm{KSub}(Y)$-is in general not preserved under substitution. Also, negation/orthocomplementation $(-)^{\perp}$ does not commute with substitution, because $1=0^{\perp}$ and $f^{-1}(1)=1$.

Being able to take pullbacks of kernels has some important consequences. 
Lemma 3 Kernels are closed under composition-and hence cokernels are, too.

Proof We shall prove the result for cokernels, because it uses pullback results as we have just seen. So assume we have (composable) cokernels $e, d$; we wish to show $e \circ d=\operatorname{coker}(\operatorname{ker}(e \circ d))$. We first notice, using Lemma 2,

$$
\operatorname{ker}(e \circ d)=\operatorname{ker}(\operatorname{coker}(\operatorname{ker}(e)) \circ d)=d^{-1}(\operatorname{ker}(e)),
$$

yielding a pullback:

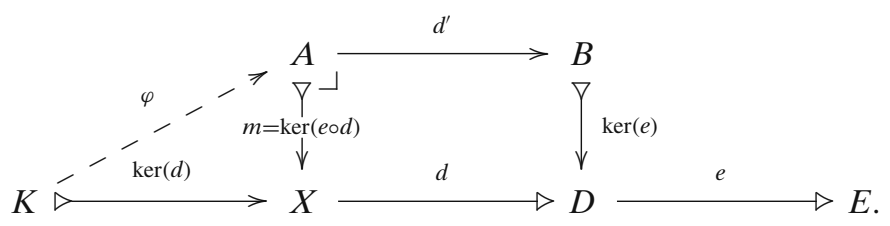

We intend to prove $e \circ d=\operatorname{coker}(m)$. Clearly, $e \circ d \circ m=e \circ \operatorname{ker}(e) \circ d^{\prime}=0 \circ$ $d^{\prime}=0$. And if $f: X \rightarrow Y$ satisfies $f \circ m=0$, then $f \circ \operatorname{ker}(d)=f \circ m \circ \varphi=0$, so because $d=$ coker $(\operatorname{ker}(d))$ there is $f^{\prime}: D \rightarrow Y$ with $f^{\prime} \circ d=f$. But then: $f^{\prime} \circ$ $\operatorname{ker}(e) \circ d^{\prime}=f^{\prime} \circ d \circ m=f \circ m=0$. Then $f^{\prime} \circ \operatorname{ker}(e)=0$, because $d^{\prime}$ is dagger epi because $d$ is, see Lemma 2. This finally yields $f^{\prime \prime}: E \rightarrow Y$ with $f^{\prime \prime} \circ e=f^{\prime}$. Hence $f^{\prime \prime} \circ e \circ d=f$.

As a result, the logic of kernels has intersections, preserved by substitution. More precisely, the indexed category $\mathrm{KSub}(-)$ from Corollary 1 is actually a functor KSub: $\mathbf{D}^{\text {op }} \rightarrow$ MSL to the category MSL of meet semi-lattices. Each poset $\operatorname{KSub}(X)$ also has disjunctions, by $m \vee n=\left(m^{\perp} \wedge n^{\perp}\right)^{\perp}$, but they are not preserved under substitution/pullback $f^{-1}$. Nevertheless, $m \vee m^{\perp}=\left(m^{\perp} \wedge m^{\perp \perp}\right)^{\perp}=\left(m^{\perp} \wedge m\right)^{\perp}=$ $0^{\perp}=1$.

The essence of the following result goes back to [9].

Proposition 1 Orthomodularity holds: for kernels $m \leq n$, say via $\varphi$ with $n \circ \varphi=m$, one has pullbacks:

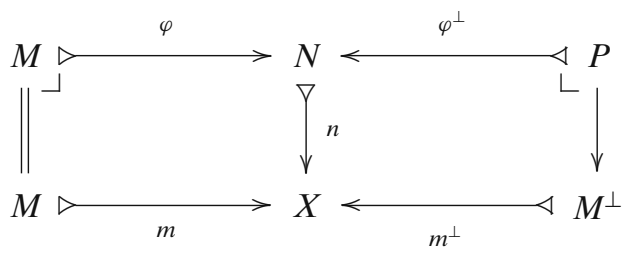

This means that $m \vee\left(m^{\perp} \wedge n\right)=n$. 
Proof The square on the left is obviously a pullback. For the one on the right we use a simple calculation, following Lemma 2:

$$
\begin{aligned}
n^{-1}\left(m^{\perp}\right) & =\operatorname{ker}\left(\operatorname{coker}\left(m^{\perp}\right) \circ n\right) \\
& =\operatorname{ker}\left(\operatorname{coker}\left(\operatorname{ker}\left(m^{\dagger}\right)\right) \circ n\right) \\
& =\operatorname{ker}\left(m^{\dagger} \circ n\right) \quad \text { since } m^{\dagger} \text { is a cokernel } \\
& \stackrel{(*)}{=} \operatorname{ker}\left(\varphi^{\dagger}\right) \\
& =\varphi^{\perp},
\end{aligned}
$$

where the marked equation holds because $n \circ \varphi=m$, so that $\varphi=n^{\dagger} \circ n \circ \varphi=n^{\dagger} \circ m$ and thus $\varphi^{\dagger}=m^{\dagger} \circ n$. Then:

$$
m \vee\left(m^{\perp} \wedge n\right)=(n \circ \varphi) \vee\left(n \circ \varphi^{\perp}\right) \stackrel{(*)}{=} n \circ\left(\varphi \vee \varphi^{\perp}\right)=n \circ \mathrm{id}=n .
$$

The (newly) marked equation holds because $n \circ(-)$ preserves joins, since it is a left adjoint: $n \circ k \leq m$ iff $k \leq n^{-1}(m)$, for kernels $k, m$.

The following notion does not seem to have an established terminology, and therefore we introduce our own.

Definition 3 In a category with a zero object, a map $m$ is called a zero-mono if $m \circ f=0$ implies $f=0$, for any map $f$. Dually, $e$ is zero-epi if $f \circ e=0$ implies $f=0$. In diagrams we write $>\circ$ for zero-monos and $\rightarrow$ for zero-epis.

Clearly, a mono is zero-mono, since $m \circ f=0=m \circ 0$ implies $f=0$ if $m$ is monic. The following points are worth making explicit.

Lemma 4 In a dagger kernel category,

1. $m$ is a zero-mono iff $\operatorname{ker}(m)=0$ and e is a zero-epi iff $\operatorname{coker}(e)=0$;

2. $\operatorname{ker}(m \circ f)=\operatorname{ker}(f)$ if $m$ is a zero-mono, and similarly, $\operatorname{coker}(f \circ e)=\operatorname{coker}(f)$ if e is a zero-epi;

3. a kernel which is zero-epic is an isomorphism.

We shall mostly be interested in zero-epis (instead of zero-monos), because they arise in the factorisation of Section 4. In the presence of dagger equalisers, zero-epis are ordinary epis. This applies to Hilb and PInj. This fact is not really used, but is included because it gives a better understanding of the situation. A dagger equaliser category is a dagger category that has equalisers which are dagger monic.

Lemma 5 In a dagger equaliser category $\mathbf{D}$ where every dagger mono is a kernel, zeroepis in $\mathbf{D}$ are ordinary epis. 
Proof Assume a zero-epi $e: E \rightarrow X$ with two maps $f, g: X \rightarrow Y$ satisfying $f \circ e=$ $g \circ e$. We need to prove $f=g$. Let $m: M \longmapsto X$ be the equaliser of $f, g$, with $h=$ coker $(m)$, as in:

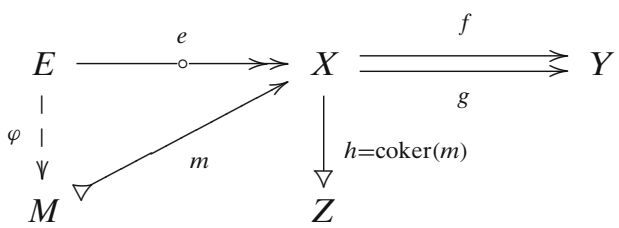

This $e$ factors through the equaliser $m$, as indicated, since $f \circ e=g \circ e$. Then $h \circ e=h \circ m \circ \varphi=0 \circ \varphi=0$. Hence $h=0$ because $e$ is zero-epi. But $m$, being a dagger mono, is a dagger kernel. Hence $m=\operatorname{ker}(\operatorname{coker}(m))=\operatorname{ker}(h)=\operatorname{ker}(0)=\mathrm{id}$, so that $f=g$.

\subsection{Indexed Categories and Fibrations}

The kernel posets $\mathrm{KSub}(X)$ capture the predicates on an object $X$, considered as underlying type, in a dagger kernel category $\mathbf{D}$. Such posets are studied systematically in categorical logic, often in terms of indexed categories $\mathbf{D}^{\text {op }} \rightarrow$ Posets or even as a socalled fibration $\left(\begin{array}{c}\operatorname{KSub}(\mathbf{D}) \\ \mathbf{D}\end{array}\right)$, see [21]. We shall occasionally borrow terminology from this setting, but will not make deep use of it. A construction that is definitely useful in the present setting is the "total" category $\operatorname{KSub}(\mathbf{D})$. It has (equivalence classes of) kernels $M \longmapsto X$ as objects. Morphisms $(M \stackrel{m}{\longmapsto} X) \longrightarrow(N \stackrel{n}{\longmapsto} Y)$ in $\operatorname{KSub}(\mathbf{D})$ are maps $f: X \rightarrow Y$ in $\mathbf{D}$ with

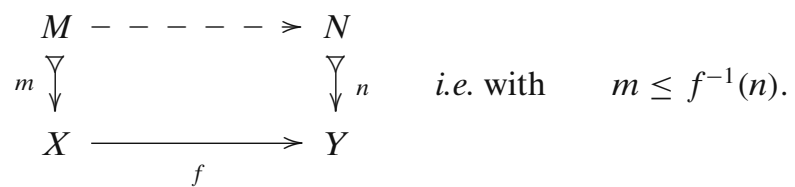

We shall sometimes refer to this fibration as the "kernel fibration". Every functor $F: \mathbf{D} \rightarrow \mathbf{E}$ in DagKerCat induces a map of fibrations:

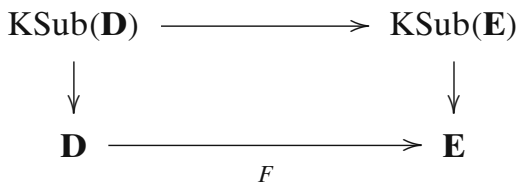

because $F$ preserves kernels and pullbacks of kernels - the latter since pullbacks can be formulated in terms of constructions that are preserved by $F$, see Lemma 2. As we shall see, in some situations, diagram (1) is a pullback-also called a change-of-base situation in this context, see [21]. This means that the map $\operatorname{KSub}(X) \rightarrow \operatorname{KSub}(F X)$ is an isomorphism.

Let us mention one result about this category $\operatorname{KSub}(\mathbf{D})$, which will be used later. 
Lemma 6 The category $\mathrm{KSub}(\mathbf{D})$ for a dagger category $\mathbf{D}$ carries an involution $\mathrm{KSub}(\mathbf{D})^{\mathrm{op}} \rightarrow \mathrm{KSub}(\mathbf{D})$ given by orthocomplementation:

$$
(M \stackrel{m}{\longmapsto} X) \longmapsto\left(M^{\perp} \stackrel{m^{\perp}}{\longmapsto} X\right) \quad \text { and } f \longmapsto f^{\dagger} \text {. }
$$

Proof The involution is well-defined because a (necessarily unique) map $\varphi$ exists if and only if a (necessarily unique) map $\psi$ exists, in commuting squares:

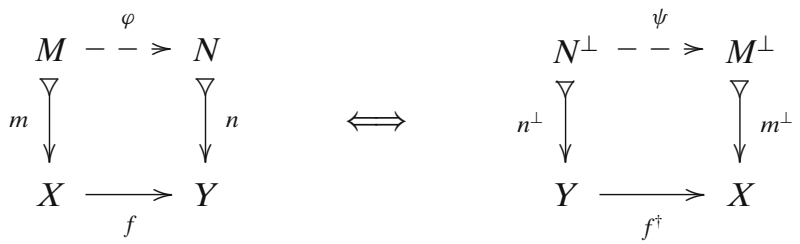

Given $\varphi$, we obtain $\psi$ because $f^{\dagger} \circ n^{\perp}$ factors through $\operatorname{ker}\left(m^{\dagger}\right)=m^{\perp}$ since

$$
m^{\dagger} \circ f^{\dagger} \circ n^{\perp}=\varphi^{\dagger} \circ n^{\dagger} \circ n^{\perp}=\varphi^{\dagger} \circ 0=0 .
$$

The reverse direction follows immediately.

\section{Main Examples}

This section describes our four main examples, namely Rel, PInj, Hilb and PHilb, and additionally a general construction to turn a Boolean algebra into a dagger kernel category.

\subsection{The Category Rel of Sets and Relations}

Sets and binary relations $R \subseteq X \times Y$ between them can be organised in the familiar category Rel, using relational composition. Alternatively, such a relation may be described as a Kleisli map $X \rightarrow \mathcal{P}(Y)$ for the powerset monad $\mathcal{P}$; in line with this representation we sometimes write $R(x)=\{y \in Y \mid R(x, y)\}$. A third way is to represent such a morphism in Rel as (an equivalence class of) a pair of maps $\left(X \stackrel{r_{1}}{\leftarrow} R \stackrel{r_{2}}{\rightarrow} Y\right.$ ) whose tuple $\left\langle r_{1}, r_{2}\right\rangle: R \rightarrow X \times Y$ of legs is injective.

There is a simple dagger on Rel, given by reversal of relations: $R^{\dagger}(y, x)=R(x, y)$. A map $R: X \rightarrow Y$ is a dagger mono in Rel if $R^{\dagger} \circ R=\mathrm{id}$, which amounts to the equivalence:

$$
\exists_{y \in Y} \cdot R(x, y) \wedge R\left(x^{\prime}, y\right) \Longleftrightarrow x=x^{\prime}
$$

for all $x, x^{\prime} \in X$. It can be split into two statements:

$$
\forall_{x \in X} \cdot \exists_{y \in Y} \cdot R(x, y) \text { and } \forall_{x, x^{\prime} \in X} \cdot \forall_{y \in Y} \cdot R(x, y) \wedge R\left(x^{\prime}, y\right) \Rightarrow x=x^{\prime} .
$$


Hence such a dagger mono $R$ is given by a span of the form

$$
\left(\begin{array}{lllll} 
& & & & \\
& r_{1} & & r_{2} \\
X & & & Y
\end{array}\right)
$$

with an surjection as first leg and an injection as second leg. A dagger epi has the same shape, but with legs exchanged.

The empty set 0 is a zero object in Rel, and the resulting zero map $0: X \rightarrow Y$ is the empty relation $\emptyset \subseteq X \times Y$.

The category Rel also has kernels. For an arbitrary map $R: X \rightarrow Y$ one takes $\operatorname{ker}(R)=\left\{x \in X \mid \neg \exists_{y \in Y} \cdot R(x, y)\right\}$ with map $k: \operatorname{ker}(R) \rightarrow X$ in Rel given by $k\left(x, x^{\prime}\right) \Leftrightarrow x=x^{\prime}$. Clearly, $R \circ k=0$. And if $S: Z \rightarrow X$ satisfies $R \circ S=0$, then $\neg \exists_{x \in X} . R(x, y) \wedge S(z, x)$, for all $z \in Z$ and $y \in Y$. This means that $S(z, x)$ implies there is no $y$ with $R(x, y)$. Hence $S$ factors through the kernel $k$. Kernels are thus of the following form:

$$
\left(\begin{array}{llll} 
& K & & \\
& & & \\
& & & X
\end{array}\right) \text { with } K=\{x \in X \mid R(x)=\emptyset\} .
$$

So, kernels are essentially given by subsets: $\mathrm{KSub}(X)=\mathcal{P}(X)$. Indeed, Rel is Boolean, in the sense of Definition 1. A cokernel has the reversed shape.

Finally, a relation $R$ is zero-mono if its kernel is 0 , see Lemma 4 . This means that $R(x) \neq \emptyset$, for each $x \in X$, so that $R$ 's left leg is a surjection.

Proposition 2 In $\mathbf{R e l}$ there are proper inclusions:

kernel $\subsetneq$ dagger mono $\subsetneq$ mono $\subsetneq$ zero-mono.

Subsets of a set $X$ correspond to kernels in $\mathbf{R e l}$ with codomain $X$.

There is of course a dual version of this result, for cokernels and epis.

Proof We still need to produce (1) a zero-mono which is not a mono, and (2) a mono which is not a dagger mono. As to (1), consider $R \subseteq\{0,1\} \times\{a, b\}$ given by $R=\{(0, a),(1, a)\}$. Its first leg is surjective, so $R$ is a zero-mono. But it is not a mono: there are two different relations $\{(*, 0)\},\{(*, 1)\} \subseteq\{*\} \times\{0,1\}$ with $R \circ\{(*, 0)\}=$ $\{(*, a)\}=R \circ\{(*, 1)\}$.

As to (2), consider the relation $R \subseteq\{0,1\} \times\{a, b, c\}$ given by $R=\{(0, a),(0, b)$, $(1, b),(1, c)\}$. Clearly, the first leg of $R$ is a surjection, and the second one is neither an injection nor a surjection. We check that $R$ is monic. Suppose $S, T: X \rightarrow\{0,1\}$ satisfy $R \circ S=R \circ T$. If $S(x, 0)$, then $(R \circ S)(x, a)=(R \circ T)(x, a)$, so that $T(x, 0)$. Similarly, $S(x, 1) \Rightarrow T(x, 1)$.

We add that the pullback $R^{-1}(n)$ of a kernel $n=(N=N \longmapsto Y)$ along a relation $R \subseteq X \times Y$, as described in Lemma 2, is the subset of $X$ given by the modal formula $\square_{R}(n)(x)=R^{-1}(n)(x) \Leftrightarrow\left(\forall_{y} . R(x, y) \Rightarrow N(y)\right)$. As is well-known in modal logic, 
$\square_{R}$ preserves conjunctions, but not disjunctions. Interestingly, the familiar "graph" functor $\mathcal{G}:$ Sets $\rightarrow$ Rel, mapping a set to itself and a function to its graph relation, yields a map of fibrations

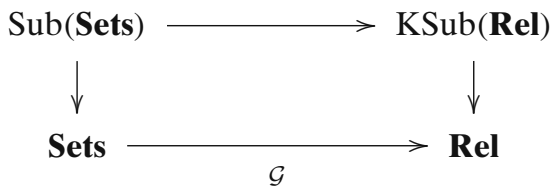

which in fact forms a pullback (or a "change-of-base" situation, see [21]). This means that the familiar logic of sets can be obtained from this kernel logic on relations. In this diagram we use that inverse image is preserved: for a function $f: X \rightarrow Y$ and predicate $N \subseteq Y$ one has:

$$
\begin{aligned}
\mathcal{G}(f)^{-1}(N)=\square_{\mathcal{G}(f)}(N) & =\left\{x \in X \mid \forall_{y} \cdot \mathcal{G}(f)(x, y) \Rightarrow N(y)\right\} \\
& =\left\{x \in X \mid \forall_{y} \cdot f(x)=y \Rightarrow N(y)\right\} \\
& =\{x \in X \mid N(f(x))\} \\
& =f^{-1}(N) .
\end{aligned}
$$

\subsection{The Category PInj of Sets and Partial Injections}

There is a subcategory PInj of Rel also with sets as objects but with "partial injections" as morphisms. These are special relations $F \subseteq X \times Y$ satisfying $F(x, y) \wedge$ $F\left(x, y^{\prime}\right) \Rightarrow y=y^{\prime}$ and $F(x, y) \wedge F\left(x^{\prime}, y\right) \Rightarrow x=x^{\prime}$. We shall therefore often write morphisms $f: X \rightarrow Y$ in PInj as spans with the notational convention

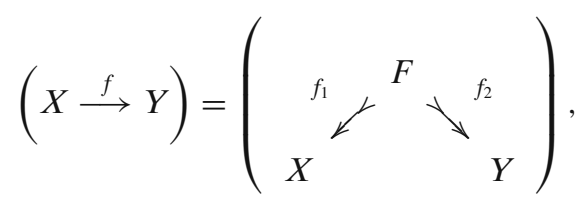

where spans $\left(X \stackrel{f_{1}}{\longleftarrow} F \stackrel{f_{2}}{\longmapsto} Y\right)$ and $\left(X \stackrel{g_{1}}{\longleftarrow} G \stackrel{g_{2}}{\longmapsto} Y\right.$ ) are equivalent if there is an isomorphism $\varphi: F \rightarrow G$ with $g_{i} \circ \varphi=f_{i}$, for $i=1,2$-like for relations.

Composition of $X \stackrel{f}{\rightarrow} Y \stackrel{g}{\rightarrow} Z$ can be described as relational composition, but also via pullbacks of spans. The identity map $X \rightarrow X$ is given by the span of identities $X \overleftrightarrow{\longleftarrow} X \longmapsto X$. The involution is inherited from Rel and can be described as $\left(X \stackrel{f_{1}}{\longleftrightarrow} F \stackrel{f_{2}}{\longmapsto} Y\right)^{\dagger}=\left(Y \stackrel{f_{2}}{\longleftrightarrow} F \stackrel{f_{1}}{\longmapsto} X\right)$.

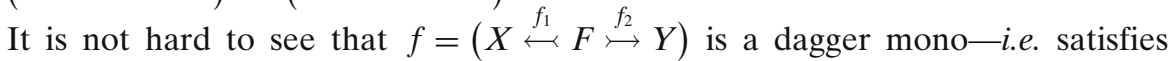
$f^{\dagger} \circ f=\mathrm{id}$-if and only if its first leg $f_{1}: F \longmapsto X$ is an isomorphism. For convenience we therefore identify a mono/injection $m: M \longmapsto X$ in Sets with the corresponding dagger mono $(M \stackrel{\text { id }}{\longleftarrow} M \stackrel{m}{\longmapsto} X)$ in PInj.

By duality: $f$ is dagger epi iff $f^{\dagger}$ is dagger mono iff the second leg $f_{2}$ of $f$ is an isomorphism. Further, $f$ is a dagger iso iff $f$ is both dagger mono and dagger epi iff both legs $f_{1}$ and $f_{2}$ of $f$ are isomorphisms.

Like in Rel, the empty set is a zero object, with corresponding zero map given by the empty relation, and $0^{\dagger}=0$. 
For the description of the kernel of an arbitrary map $f=\left(X \stackrel{f_{1}}{\longleftarrow} F \stackrel{f_{2}}{\longmapsto} Y\right)$ in PInj we shall use the ad hoc notation $\neg_{1} F \stackrel{\neg f_{1}}{\longmapsto} X$ for the negation of the first leg $f_{1}: F \longmapsto X$, as subobject/subset. It yields a map:

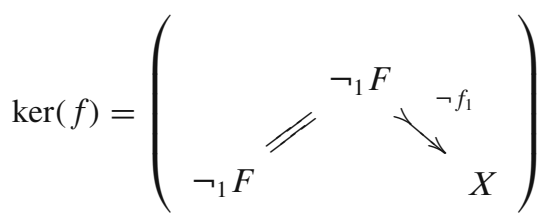

It satisfies $f \circ \operatorname{ker}(f)=0$. It is a dagger mono by construction. Notice that kernels are the same as dagger monos, and are also the same as zero-monos. They all correspond to subsets, so that $\operatorname{KSub}(X)=\mathcal{P}(X)$ and PInj is Boolean, like Rel.

The next result summarises what we have seen so far and shows that PInj is very different from Rel (see Proposition 2).

Proposition 3 In PInj there are proper identities:

$$
\text { kernel }=\text { dagger mono }=\text { mono }=\text { zero-mono. }
$$

These all correspond to subsets.

\subsection{The Category Hilb of Hilbert Spaces}

Our third example is the category Hilb of (complex) Hilbert spaces and continuous linear maps. Recall that a Hilbert space is a vector space $X$ equipped with an inner product, i.e. a function $\langle-\mid-\rangle: X \times X \rightarrow \mathbb{C}$ that is linear in the first and anti-linear in the second variable, satisfies $\langle x \mid x\rangle \geq 0$ with equality if and only if $x=0$, and $\langle x \mid y\rangle=$ $\overline{\langle y \mid x\rangle}$. Moreover, a Hilbert space must be complete in the metric induced by the inner product by $d(x, y)=\sqrt{\langle x-y \mid x-y\rangle}$.

The Riesz representation theorem provides this category with a dagger. Explicitly, for $f: X \rightarrow Y$ a given morphism, $f^{\dagger}: Y \rightarrow X$ is the unique morphism satisfying

$$
\langle f(x) \mid y\rangle_{Y}=\left\langle x \mid f^{\dagger}(y)\right\rangle_{X}
$$

for all $x \in X$ and $y \in Y$. The zero object is inherited from the category of (complex) vector spaces: it is the zero-dimensional Hilbert space $\{0\}$, with unique inner product $\langle 0 \mid 0\rangle=0$.

In the category Hilb, dagger mono's are usually called isometries, because they preserve the metric: $f^{\dagger} \circ f=$ id if and only if

$$
d(f x, f y)=\langle f(x-y) \mid f(x-y)\rangle^{\frac{1}{2}}=\left\langle x-y \mid\left(f^{\dagger} \circ f\right)(x-y)\right\rangle^{\frac{1}{2}}=d(x, y) .
$$

Kernels are inherited from the category of vector spaces. For $f: X \rightarrow Y$, we can choose $\operatorname{ker}(f)$ to be (the inclusion of) $\{x \in X \mid f(x)=0\}$, as this is complete with respect to the restricted inner product of $X$. Hence kernels correspond to (inclusions of) closed subspaces. Being inclusions, kernels are obviously dagger monos. Hence Hilb is indeed an example of a dagger kernel category. However, Hilb is not Boolean. The following proposition shows that it is indeed different, categorically, from Rel and PInj. 
Proposition 4 In Hilb one has:

$$
\text { kernel }=\text { dagger mono } \subsetneq \text { mono }=\text { zero-mono. }
$$

Proof For the left equality, notice that both kernels and isometries correspond to closed subspaces. It is not hard to show that the monos in Hilb are precisely the injective continuous linear functions, establishing the middle proper inclusion. Finally, Hilb has equalisers by eq $(f, g)=\operatorname{ker}(g-f)$, which takes care of the right equality.

As is well-known, the $\ell^{2}$ construction forms a functor $\ell^{2}:$ PInj $\rightarrow$ Hilb (but not a functor Sets $\rightarrow$ Hilb), see e.g. [3, 15]. Since it preserves daggers, zero object and kernels it is a map in the category DagKerCat, and therefore yields a map of kernel fibrations like in (1). It does not form a pullback (change-of-base) between these fibrations, since the map $\operatorname{KSub}_{\mathbf{P I n j}}(X)=\mathcal{P}(X) \rightarrow \operatorname{KSub}_{\mathbf{H i l b}}\left(\ell^{2}(X)\right)$ is not an isomorphism.

\subsection{The Category PHilb: Hilbert Spaces Modulo Phase}

The category PHilb of projective Hilbert spaces has the same objects as Hilb, but its homsets are quotiented by the action of the circle group $U(1)=\{z \in \mathbb{C}|| z \mid=1\}$. That is, continuous linear transformations $f, g: X \rightarrow Y$ are identified when $x=z \cdot y$ for some phase $z \in U(1)$.

Equivalently, we could write $P X=X_{1} / U(1)$ for an object of PHilb, where $X \in$ Hilb and $X_{1}=\{x \in X \mid\|x\|=1\}$. Two vectors $x, y \in X_{1}$ are therefore identified when $x=z \cdot y$ for some $z \in U(1)$. Continuous linear transformations $f, g: X \rightarrow Y$ then descend to the same function $P X \rightarrow P Y$ precisely when they are equivalent under the action of $U(1)$. This gives a full functor $P:$ Hilb $\rightarrow$ PHilb.

The dagger of Hilb descends to PHilb, because if $f=z \cdot g$ for some $z \in U(1)$, then

$$
\langle f(x) \mid y\rangle=\bar{z} \cdot\langle g(x) \mid y\rangle=\bar{z} \cdot\left\langle x \mid g^{\dagger}(y)\right\rangle=\left\langle x \mid \bar{z} \cdot g^{\dagger}(y)\right\rangle,
$$

whence also $f^{\dagger}=\bar{z} \cdot g^{\dagger}$, making the dagger well-defined.

Also dagger kernels in Hilb descend to PHilb. More precisely, the kernel $\operatorname{ker}(f)=$ $\{x \in X \mid f(x)=0\}$ of a morphism $f: X \rightarrow Y$ is well-defined, for if $f=z \cdot f^{\prime}$ for some $z \in U(1)$, then

$$
\operatorname{ker}(f)=\left\{x \in X \mid z \cdot f^{\prime}(x)=0\right\}=\left\{x \in X \mid f^{\prime}(x)=0\right\}=\operatorname{ker}\left(f^{\prime}\right) .
$$

\section{Proposition 5 In PHilb one has:}

$$
\text { kernel }=\text { dagger mono } \subsetneq \text { mono }=\text { zero-mono } .
$$

Proof It remains to be shown that every zero-mono is a mono. So let $m: Y \rightarrow Z$ be a zero-mono, and $f, g: X \rightarrow Y$ arbitrary morphisms in PHilb. More precisely, let $m, f$ and $g$ be morphisms in Hilb representing the equivalence classes $[m],[f]$ and $[g]$ that are morphisms in PHilb. Suppose that $[m \circ f]=[m \circ g]$. Then $m \circ f \sim m \circ g$, say $m \circ f=z \cdot(m \circ g)$ for $z \in U(1)$. So $m \circ(f-z \cdot g)=0$, and $f-z \cdot g=0$ since $m$ is zero-mono. Then $f=z \cdot g$ and hence $f \sim g$, i.e. $[f]=[g]$. Thus $m$ is mono. 
The full functor $P$ : Hilb $\rightarrow$ PHilb preserves daggers, the zero object and kernels. Hence it is a map in the category DagKerCat. In fact it yields a pullback (change-ofbase) between the corresponding kernel fibrations.

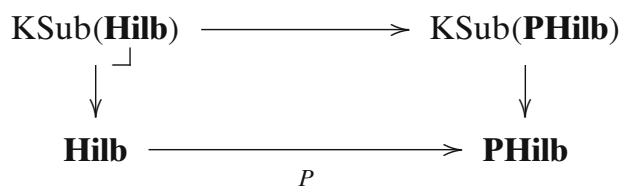

\subsection{From Boolean Algebras to Dagger Kernel Categories}

The previous four examples were concrete categories, to which we add a generic construction turning an arbitrary Boolean algebra into a (Boolean) dagger kernel category.

To start, let $B$ with $(1, \wedge)$ be a meet semi-lattice. We can turn it into a category, for which we use the notation $\widehat{B}$. The objects of $\widehat{B}$ are elements $x \in B$, and its morphisms $x \rightarrow y$ are elements $f \in B$ with $f \leq x$, y, i.e. $f \leq x \wedge y$. There is an identity $x: x \rightarrow$ $x$, and composition of $f: x \rightarrow y$ and $g: y \rightarrow z$ is simply $f \wedge g: x \rightarrow z$. This $\widehat{B}$ is a dagger category with $f^{\dagger}=f$. A map $f: x \rightarrow y$ is a dagger mono if $f^{\dagger} \circ f=f \wedge$ $f=x$. Hence a dagger mono is of the form $x: x \rightarrow y$ where $x \leq y$.

It is not hard to see that the construction $B \mapsto \widehat{B}$ is functorial: a morphism $h: B \rightarrow C$ of meet semi-lattices yields a functor $\widehat{h}: \widehat{B} \rightarrow \widehat{C}$ by $x \mapsto h(x)$. It clearly preserves $\dagger$.

Proposition 6 If $B$ is a Boolean algebra, then $\widehat{B}$ is a Boolean dagger kernel category. This yields a functor $\mathbf{B A} \rightarrow$ DagKerCat.

Proof The bottom element $0 \in B$ yields a zero object $0 \in \widehat{B}$, and also a zero map $0: x \rightarrow y$. For an arbitrary map $f: x \rightarrow y$ there is a kernel $\operatorname{ker}(f)=\neg f \wedge x$, which is a dagger mono $\operatorname{ker}(f): \operatorname{ker}(f) \rightarrow x$ in $\widehat{B}$. Clearly, $f \circ \operatorname{ker}(f)=f \wedge \neg f \wedge x=0 \wedge$ $x=0$. If also $g: z \rightarrow x$ satisfies $f \circ g=0$, then $g \leq x, z$ and $f \wedge g=0$. The latter yields $g \leq \neg f$ and thus $g \leq \neg f \wedge x=\operatorname{ker}(f)$. Hence $g$ forms the required mediating map $g: z \rightarrow \operatorname{ker}(f)$ with $\operatorname{ker}(f) \circ g=g$.

Notice that each dagger mono $m: m \rightarrow x$, where $m \leq x$, is a kernel, namely of its cokernel $\neg m \wedge x: x \rightarrow(\neg m \wedge x)$. For two kernels $m: m \rightarrow x$ and $n: n \rightarrow x$, where $m, n \leq x$, one has $m \leq n$ as kernels iff $m \leq n$ in $B$. Thus $\operatorname{KSub}(x)=\downarrow x$, which is again a Boolean algebra (with negation $\neg x=\neg m \wedge x$ ). The intersection $m \wedge n$ as subobjects is the meet $m \wedge n$ in $B$. This allows us to show that $\widehat{B}$ is Boolean: if $m \wedge$ $n=0$, them $m^{\dagger} \circ n=m \circ n=m \wedge n=0$.

The straightforward extension of the above construction to orthomodular lattices does not work: in order to get kernels one needs to use the and-then connective ( \&, see Proposition 9) for composition; but \& is neither associative nor commutative, unless the lattice is Boolean [30]. However, at the end of [22] a dagger kernel category is constructed out of an orthomodular lattice in a different manner, namely via the (dagger) Karoubi envelope of the associated Foulis semigroup. For more 
information about orthomodular lattices, see [26], and for general constructions, see for instance [16].

\section{Factorisation}

In this section we assume that $\mathbf{D}$ is an arbitrary dagger kernel category. We will show that each map in $\mathbf{D}$ can be factored as a zero-epi followed by a kernel, in an essentially unique way. This factorisation leads to existential quantifiers $\exists$, as is standard in categorical logic.

The image of a morphism $f: X \rightarrow Y$ is defined as $\operatorname{ker}(\operatorname{coker}(f))$. Since it is defined as a kernel, an image is really an equivalence class of morphisms with codomain $X$, up to isomorphism of the domain. We denote a representing morphism by $i_{f}$, and its domain by $\operatorname{Im}(f)$. As with kernels, we can choose $i_{f}$ to be dagger mono. Both the morphism $i_{f}$ and the object $\operatorname{Im}(f)$ are referred to as the image of $f$. Explicitly, it can be obtained in the following steps. First take the kernel $k$ of $f^{\dagger}$ :

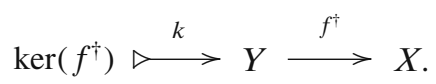

Then define $i_{f}$ as the kernel of $k^{\dagger}$, as in the following diagram:

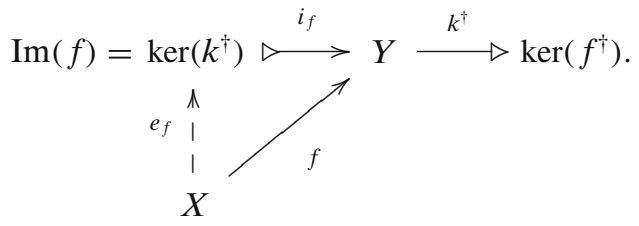

The map $e_{f}: X \rightarrow \operatorname{Im}(f)$ is obtained from the universal property of kernels, since $k^{\dagger} \circ f=\left(f^{\dagger} \circ k\right)^{\dagger}=0^{\dagger}=0$. Since $i_{f}$ was chosen to be dagger mono, this $e_{f}$ is determined as $e_{f}=\mathrm{id} \circ e_{f}=\left(i_{f}\right)^{\dagger} \circ i_{f} \circ e_{f}=\left(i_{f}\right)^{\dagger} \circ f$.

So images are defined as dagger kernels. Conversely, every dagger kernel $m=$ $\operatorname{ker}(f)$ arises as an image, since $\operatorname{ker}(\operatorname{coker}(m))=m$ by Lemma 1.

The maps that arise as $e_{f}$ in (6) can be characterised.

Proposition $\mathbf{7}$ The maps in $\mathbf{D}$ that arise of the form $e_{f}$, as in diagram (6), are precisely the zero-epis.

Proof We first show that $e_{f}$ is a zero-epi. So, assume a map $h: \operatorname{ker}\left(k^{\dagger}\right) \rightarrow Z$ satisfying $h \circ e_{f}=0$. Recall that $e_{f}=\left(i_{f}\right)^{\dagger} \circ f$, so that:

$$
f^{\dagger} \circ\left(i_{f} \circ h^{\dagger}\right)=\left(h \circ\left(i_{f}\right)^{\dagger} \circ f\right)^{\dagger}=\left(h \circ e_{f}\right)^{\dagger}=0^{\dagger}=0 .
$$

This means that $i_{f} \circ h^{\dagger}$ factors through the kernel of $f^{\dagger}$, say via $a: Z \rightarrow \operatorname{ker}\left(f^{\dagger}\right)$ with $k \circ a=i_{f} \circ h^{\dagger}$. Since $k$ is a dagger mono we now get:

$$
a=k^{\dagger} \circ k \circ a=k^{\dagger} \circ i_{f} \circ h^{\dagger}=0 \circ h^{\dagger}=0 .
$$

But then $i_{f} \circ h^{\dagger}=k \circ a=k \circ 0=0=i_{f} \circ 0$, so that $h^{\dagger}=0$, because $i_{f}$ is mono, and $h=0$, as required. 
Conversely, assume $g: X \rightarrow Y$ is a zero-epi, so that $\operatorname{coker}(g)=0$ by Lemma 4 . Trivially, $i_{g}=\operatorname{ker}(\operatorname{coker}(g))=\operatorname{ker}(X \rightarrow 0)=\mathrm{id}_{X}$, so that $e_{g}=g$.

The factorisation $f=i_{f} \circ e_{f}$ from (6) describes each map as a zero-epi followed by a kernel. In fact, these zero-epis and kernels also satisfy what is usually called the "diagonal fill-in" property.

Lemma 7 In any commuting square of shape

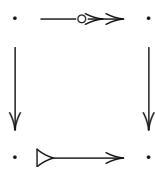

there is a (unique) diagonal

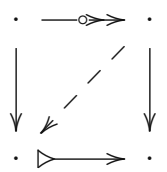

making both triangles commute.

As a result, the factorisation (6) is unique up to isomorphism. Indeed, kernels and zero-epis form a factorisation system (see [4]).

Proof Assume the zero-epi $e: E \rightarrow Y$ and kernel $m=\operatorname{ker}(h): M \longmapsto X$ satisfy $m \circ$ $f=g \circ e$, as below,

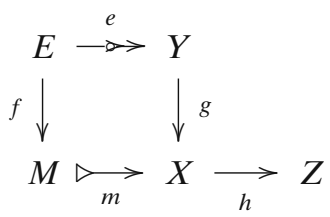

Then: $h \circ g \circ e=h \circ m \circ f=0 \circ f=0$ and $h \circ g=0$ because $e$ is zero-epi. This yields the required diagonal $d: Y \rightarrow M$ with $m \circ d=g$ because $m$ is the kernel of $h$. Using that $m$ is monic we get $d \circ e=f$.

Factorisation standardly gives a left adjoint to inverse image (pullback), corresponding to existential quantification in logic. In this self-dual situation there are alternative descriptions.

Notice that this general prescription of quantifiers by categorical logic, when applied to our quantum setting, is of a different nature from earlier attempts at quantifiers for quantum logic $[23,36]$, as it concerns multiple orthomodular lattices instead of a single one.

Proposition 8 For $f: X \rightarrow Y$, the pullback functor $f^{-1}: \operatorname{KSub}(Y) \rightarrow \operatorname{KSub}(X)$ from Lemma 2 has a left adjoint $\exists_{f}$ given as image:

$$
\left(M \triangleright^{m} \rightarrow X\right) \longmapsto\left(\operatorname{Im}(f \circ m) \triangleright^{\exists_{f}(m)=i_{f \circ m}} \longrightarrow Y\right)
$$

Alternatively, $\exists_{f}(m)=\left(\left(f^{\dagger}\right)^{-1}\left(m^{\perp}\right)\right)^{\perp}$. 
Proof The heart of the matter is that in the following diagram, the map $\varphi$ (uniquely) exists if and only if the map $\psi$ (uniquely) exists:

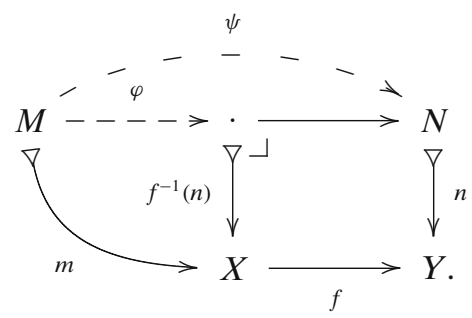

Thus one easily reads off:

$$
\begin{aligned}
m \leq f^{-1}(n) & \Longleftrightarrow \text { there is } \varphi \text { such that } m=f^{-1}(n) \circ \varphi \\
& \Longleftrightarrow \text { there is } \psi \text { such that } f \circ m=n \circ \psi \\
& \Longleftrightarrow \exists_{f}(m) \leq n .
\end{aligned}
$$

For the alternative description:

This adjunction $\exists_{f} \dashv f^{-1}$ makes the kernel fibration $\left(\begin{array}{c}\operatorname{KSub}(\mathbf{D}) \\ \downarrow \\ \mathbf{D}\end{array}\right)$ an opfibration, and thus a bifibration, see [21]. Recall the Beck-Chevalley condition: if the left square below is a pullback in $\mathbf{D}$, then the right one must commute.

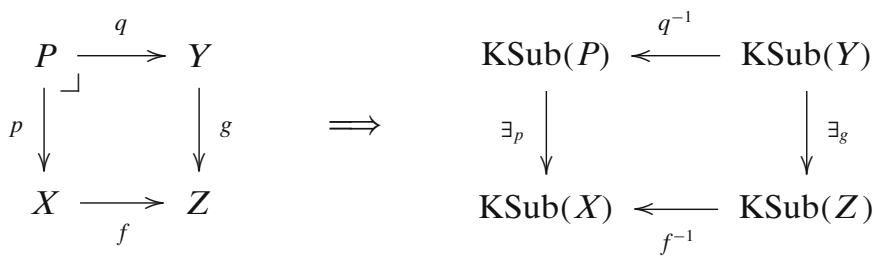

This condition ensures that $\exists$ commutes with substitution. If one restricts attention to the pullbacks of the form given in Lemma 2, then Beck-Chevalley holds. In the notation of Lemma 2, for kernels $k: K \longmapsto Y$ and $g$ : $Y \longmapsto Z$ :

$$
\begin{aligned}
f^{-1}\left(\exists_{g}(k)\right) & =f^{-1}(g \circ k) \text { because both } g, k \text { are kernels } \\
& =p \circ q^{-1}(k) \text { by composition of pullbacks } \\
& =\exists_{p}\left(q^{-1}(k)\right) .
\end{aligned}
$$

In Hilb all pullbacks exist and Beck-Chevalley holds for all of them by [5, II, Proposition 1.7.6] using Hilb's biproducts and equalisers.

The final result in this section brings more clarity; it underlies the relations between the various maps in the propositions in the previous section.

Lemma 8 If zero-epis are (ordinary) epis, then dagger monos are kernels.

Recall that Lemma 5 tells that zero-epis are epis in the presence of equalisers. 
Proof Suppose $m: M \longmapsto X$ is a dagger mono, with factorisation $m=i \circ e$ as in (6), where $i$ is a kernel and a dagger mono, and $e$ is a zero-epi and hence an epi by assumption. We are done if we can show that $e$ is an isomorphism. Since $m=i \circ e$ and $i$ is dagger monic we get $i^{\dagger} \circ m=i^{\dagger} \circ i \circ e=e$. Hence $e^{\dagger} \circ e=\left(i^{\dagger} \circ m\right)^{\dagger} \circ e=m^{\dagger} \circ$ $i \circ e=m^{\dagger} \circ m=$ id because $m$ is dagger mono. But then also $e \circ e^{\dagger}=$ id because $e$ is epi and $e \circ e^{\dagger} \circ e=e$.

Example 1 In the category Rel the image of a morphism $\left(X \stackrel{r_{1}}{\leftarrow} R \stackrel{r_{2}}{\rightarrow} Y\right)$ is the relation $i_{R}=\left(Y^{\prime} \stackrel{=}{\leftarrow} Y^{\prime} \longmapsto Y\right)$ where $Y^{\prime}=\left\{y \in Y \mid \exists_{x} . R(x, y)\right\}$ is the image of the second leg $r_{2}$ in Sets. The associated zero-epi is $e_{R}=\left(X \stackrel{r_{1}}{\leftarrow} R \stackrel{r_{2}}{\rightarrow} Y^{\prime}\right)$. Existential quantification $\exists_{R}(M)$ from Proposition 8 corresponds to the modal diamond operator (for the reversed relation $R^{\dagger}$ ):

$$
\exists_{R}(M)=\left\{y \in Y \mid \exists_{x \in M} . R(x, y)\right\}=\diamond_{R^{\dagger}}(M)=\neg \square_{R^{\dagger}}(\neg M) .
$$

It is worth mentioning that the "graph" map of fibrations (4) between sets and relations is also a map of opfibrations: for a function $f: X \rightarrow Y$ and a predicate $M \subseteq X$ one has:

$$
\begin{aligned}
\exists_{\mathcal{G}(f)}(M) & =\left\{y \mid \exists_{x} \cdot \mathcal{G}(f)(x, y) \wedge M(x)\right\} \\
& =\left\{y \mid \exists_{x} . f(x)=y \wedge M(x)\right\} \\
& =\{f(x) \mid M(x)\} \\
& =\exists_{f}(M),
\end{aligned}
$$

where $\exists_{f}$ in the last line is the left adjoint to pullback $f^{-1}$ in the category Sets.

In PInj the image of a map $f=\left(X \stackrel{f_{1}}{\longleftarrow} F \stackrel{f_{2}}{\longmapsto} Y\right)$ is given as $i_{f}=\left(F \stackrel{\text { id }}{\longleftarrow} F \stackrel{f_{2}}{\longmapsto} Y\right)$. The associated map $e_{f}$ is $\left(X \stackrel{f_{1}}{\longleftarrow} F \stackrel{\text { id }}{\longmapsto} F\right)$, so that indeed $f=i_{f} \circ e_{f}$. Notice that this $e_{f}$ is a dagger epi in PInj.

In Hilb, the image of a map $f: X \rightarrow Y$ is (the inclusion of) the closure of the set-theoretic image $\left\{y \in Y \mid \exists_{x \in X} \cdot y=f(x)\right\}$. This descends to PHilb: the image of a morphism is the equivalence class represented by the inclusion of the closure of the set-theoretic image of a representative.

The functor $\ell^{2}:$ PInj $\rightarrow$ Hilb is a map of opfibrations: for a partial injection $f=$ $\left(X \stackrel{f_{1}}{\longleftarrow} F \stackrel{f_{2}}{\longmapsto} Y\right)$ and a kernel $m: M \longmapsto X$ in PInj one has:

$$
\begin{aligned}
\exists_{\ell^{2}(f)}\left(\ell^{2}(m)\right) & =\operatorname{Im}_{\text {Hilb }}\left(\ell^{2}(f \circ m)\right) \\
& \left.=\operatorname{Im}_{\text {Hilb }}\left(\ell^{2}(M) \times Y \ni(\varphi, y) \mapsto \sum_{x \in(f \circ m)^{-1}(y)} \varphi(x)\right)\right) \\
& \cong \overline{\left\{\varphi \in \ell^{2}(X) \mid \operatorname{supp}(\varphi) \subseteq F \cap M\right\}} \\
& =\left\{\varphi \in \ell^{2}(X) \mid \operatorname{supp}(\varphi) \subseteq F \cap M\right\} \\
& \cong \ell^{2}\left(f_{2} \circ f_{1}^{-1}(m)\right) \\
& =\ell^{2}\left(\exists_{f}(m)\right) .
\end{aligned}
$$


Also the full functor $P:$ Hilb $\rightarrow$ PHilb is a map of opfibrations: for $f: X \rightarrow Y$ and a kernel $m: M \longmapsto X$ in Hilb one has:

$$
\begin{aligned}
\exists_{P f}(P m) & =\operatorname{Im}_{\text {PHilb }}(P(f \circ m)) \\
& =\overline{\{f(x) \mid x \in M\}} \\
& =P(\overline{\{f(x) \mid x \in M\}}) \\
& =P\left(\operatorname{Im}_{\text {Hilb }}(f \circ m)\right) \\
& =P\left(\exists_{f}(m)\right) .
\end{aligned}
$$

In the category $\widehat{B}$ obtained from a Boolean algebra the factorisation of $f: x \rightarrow y$ is the composite $x \stackrel{f}{\longrightarrow} f \stackrel{f}{\longrightarrow} y$. In particular, for $m \leq x$, considered as kernel $m: m \rightarrow x$ one has $\exists_{f}(m)=(m \wedge f:(m \wedge f) \rightarrow x)$.

Example 2 In [33] the domain $\operatorname{Dom}(f)$ of a map $f: X \rightarrow Y$ is the complement of its kernel, so $\operatorname{Dom}(f)=\operatorname{ker}(f)^{\perp}$, and hence a kernel itself. It can be described as an image, namely of $f^{\dagger}$, since:

$$
\operatorname{Dom}(f)=\operatorname{ker}(f)^{\perp}=\operatorname{ker}\left(\operatorname{ker}(f)^{\dagger}\right)=\operatorname{ker}\left(\operatorname{coker}\left(f^{\dagger}\right)\right)=i_{f^{\dagger}} .
$$

It is shown in [33] that the composition $f \circ \operatorname{Dom}(f)$ is zero-monic-or "total", as it is called there. This also holds in the present setting, since:

$$
f \circ \operatorname{Dom}(f)=f^{\dagger \dagger} \circ i_{f^{\dagger}}=\left(i_{f^{\dagger}} \circ e_{f^{\dagger}}\right)^{\dagger} \circ i_{f^{\dagger}}=\left(e_{f^{\dagger}}\right)^{\dagger} \circ\left(i_{f^{\dagger}}\right)^{\dagger} \circ i_{f^{\dagger}}=\left(e_{f^{\dagger}}\right)^{\dagger} .
$$

This $e_{f^{\dagger}}$ is zero-epic, by Proposition 7, so that $\left(e_{f^{\dagger}}\right)^{\dagger}$ is indeed zero-monic. In case $f: X \rightarrow X$ is a self-adjoint map, meaning $f^{\dagger}=f$, then the image of $f$ is the same as the domain, and thus as the complement of the kernel.

There is one further property that is worth making explicit, if only in examples. In the kernel fibration over Rel one finds the following correspondences.

$$
\operatorname{KSub}(X) \cong \mathcal{P}(X) \cong \operatorname{Sets}(X, 2) \cong \operatorname{Sets}(X, \mathcal{P}(1)) \cong \operatorname{Rel}(X, 1) .
$$

This suggests that one has "kernel classifiers", comparable to "subobject classifiers" in a topos-or more abstractly, "generic objects", see [21]. But the naturality that one has in toposes via pullback functors $f^{-1}$ exists here via their left adjoints $\exists_{f}$. That is, we really have found a natural correspondence $\operatorname{KSub}(X) \cong \operatorname{Rel}(1, X)$ instead of $\operatorname{KSub}(X) \cong \boldsymbol{R e l}(X, 1)$. Indeed, there are natural "characteristic" isomorphisms:

$$
\begin{aligned}
\operatorname{KSub}(X)= & \mathcal{P}(X) \frac{\text { char }}{\cong} \\
(M \subseteq X) \longmapsto & \operatorname{Rel}(1, X) \\
& \cong\{(*, x) \mid x \in M\}
\end{aligned}
$$

Then, for $S: X \rightarrow Y$ in Rel,

$$
\begin{aligned}
S \circ \operatorname{char}(M) & =\left\{(*, y) \mid \exists_{x} \cdot \operatorname{char}(M)(*, x) \wedge S(x, y)\right\} \\
& =\left\{(*, y) \mid \exists_{x} \cdot M(x) \wedge S(x, y)\right\} \\
& =\left\{(*, y) \mid \exists_{S}(M)(y)\right\} \\
& =\operatorname{char}\left(\exists_{S}(M)\right) .
\end{aligned}
$$


Hence one could say that Rel has a kernel "opclassifier". This naturality explains our choice of $\operatorname{Rel}(1, X)$ over $\operatorname{Rel}(X, 1)$ : the latter formulation more closely resembles the subobject classifiers of a topos, but using the former, naturality can be formulated without using the dagger. Hence in principle one could even consider "opclassifiers" in categories without a dagger.

The same thing happens in the dagger categories $\widehat{B}$ from Section 3.5. There one has, for $x \in B$,

$$
\begin{aligned}
\operatorname{KSub}(x)=\downarrow x \longrightarrow & \text { char } \\
(m \leq x) \longmapsto & \widehat{B}(1, x) \\
& \longrightarrow(m: 1 \rightarrow x)
\end{aligned}
$$

As before, $f \circ \operatorname{char}(m)=f \wedge m=\exists_{f}(m)=\operatorname{char}\left(\exists_{f}(m)\right)$.

The category OMLatGal of orthomodular lattices and Galois connections between them from [22] also has such an opclassifier. There is no obvious kernel opclassifier for the category Hilb. The category PInj is easily seen not to have a kernel opclassifier.

\section{Images and Coimages}

We continue to work in an arbitrary dagger kernel category D. In the previous section we have seen how each map $f: X \rightarrow Y$ in $\mathbf{D}$ can be factored as $f=i_{f} \circ e_{f}$ where the image $i_{f}=\operatorname{ker}(\operatorname{coker}(f)): \operatorname{Im}(f) \longmapsto Y$ is a kernel and $e_{f}$ is a zero-epi. We can apply this same factorisation to the dual $f^{\dagger}$. The dual of its image, $\left(i_{f^{\dagger}}\right)^{\dagger}=$ coker $(\operatorname{ker}(f)): X \rightarrow \operatorname{Im}\left(f^{\dagger}\right)$, is commonly called the coimage of $f$. It is a cokernel and dagger epi by construction. Thus we have:
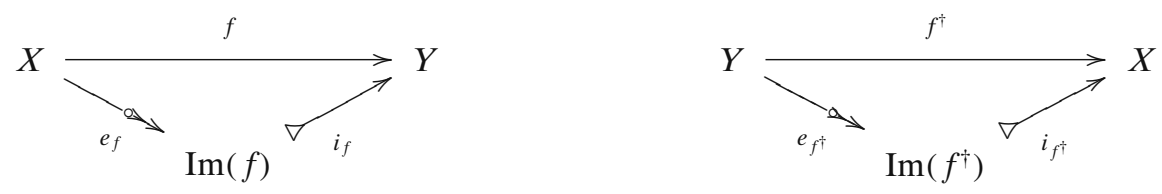

By combining these factorisations we get two mediating maps $m$ by diagonal fill-in (see Lemma 7), as in:
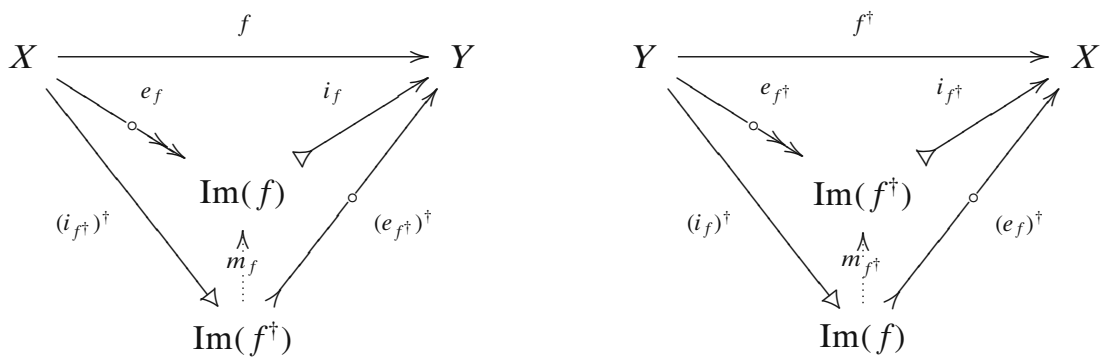
We claim that $\left(m_{f}\right)^{\dagger}=m_{f^{\dagger}}$. This follows easily from the fact that $\left(i_{f^{\dagger}}\right)^{\dagger}$ is epi:

$$
\left(m_{f^{\dagger}}\right)^{\dagger} \circ\left(i_{f^{\dagger}}\right)^{\dagger}=\left(i_{f^{\dagger}} \circ m_{f^{\dagger}}\right)^{\dagger}=\left(e_{f}\right)^{\dagger \dagger}=e_{f}=m_{f} \circ\left(i_{f^{\dagger}}\right)^{\dagger} .
$$

Moreover, $m_{f}$ is both a zero-epi and a zero-mono.

As a result we can factorise each map $f: X \rightarrow Y$ in $\mathbf{D}$ as:

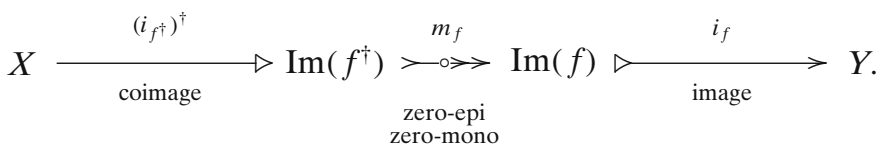

This coimage may also be reversed, so that a map in $\mathbf{D}$ can also be understood as a pair of kernels with a zero-mono/epi between them, as in:

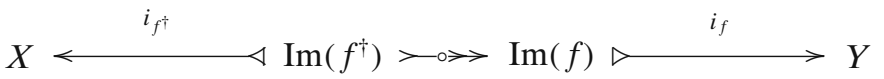

The two outer kernel maps perform some "bookkeeping" to adjust the types; the real action takes place in the middle, see the examples below. The category PInj consists, in a sense, of only these bookkeeping maps, without any action. This will be described more systematically in Definition 4.

Example 3 We briefly describe the factorisation (7) in Rel, PInj and Hilb, using diagrammatic order for convenience (with notation $f ; g=g \circ f$ ).

For a map $\left(X \stackrel{r_{1}}{\leftarrow} R \stackrel{r_{2}}{\rightarrow} Y\right.$ ) in Rel we take the images $X^{\prime} \longmapsto X$ of $r_{1}$ and $Y^{\prime} \longmapsto Y$ of $r_{2}$ in:

$$
\begin{aligned}
& \left(\begin{array}{llll} 
& & & \\
& & R & \\
& r_{1} & & r_{2} \\
X & & & Y
\end{array}\right)
\end{aligned}
$$

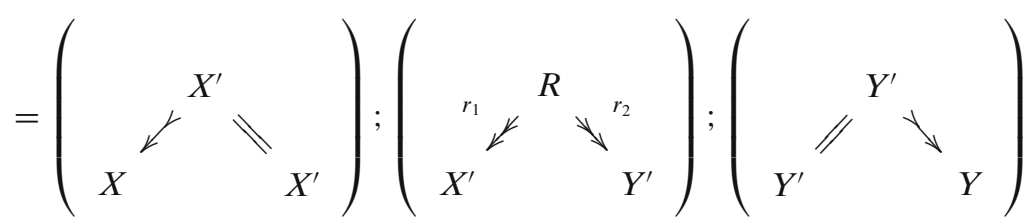

In PInj the situation is simpler, because the middle part $m$ in (7) is the identity, in:

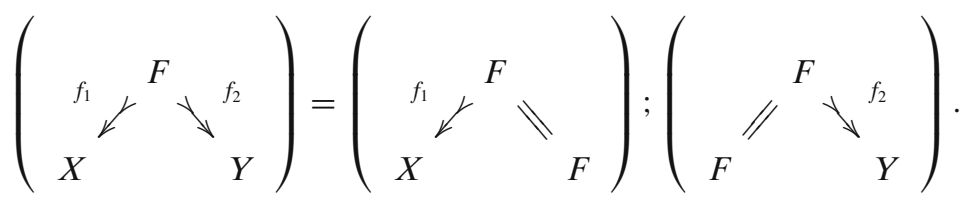

In Hilb, a morphism $f: X \rightarrow Y$ factors as $f=i \circ m \circ e$. The third part $i: I \rightarrow Y$ is given by $i(y)=y$, where $I$ is the closure $\overline{\{f(x): x \in X\}}$. The first part $e: X \rightarrow E$ 
is given by orthogonal projection on the closure $E=\overline{\left\{f^{\dagger}(y): y \in Y\right\}}$; explicitly, $e(x)$ is the unique $x^{\prime}$ such that $x=x^{\prime}+x^{\prime \prime}$ with $x^{\prime} \in E$ and $\left\langle x^{\prime \prime} \mid z\right\rangle=0$ for all $z \in E$. Using the fact that the adjoint $e^{\dagger}: E \rightarrow X$ is given by $e^{\dagger}(x)=x$, we deduce that the middle part $m: E \rightarrow I$ is determined by $m(x)=(i \circ m)(x)=\left(f \circ e^{\dagger}\right)(x)=f(x)$. Explicitly,

$$
(X \stackrel{f}{\longrightarrow} Y)=(X \stackrel{e}{\longrightarrow} E) ;(E \stackrel{m}{\longrightarrow} I) ;(I \stackrel{i}{\longrightarrow} Y) \text {. }
$$

\section{Categorical Logic}

This section further investigates the logic of dagger kernel categories. We shall first see how the so-called Sasaki hook [26] arises naturally in this setting, and then investigate Booleanness.

For a kernel $m: M \longmapsto X$ we shall write $\mathfrak{E}(m)=m \circ m^{\dagger}: X \rightarrow X$ for the "effect" of $m$, see [11]. This $\mathfrak{E}(m)$ is easily seen to be a self-adjoint idempotent: one has $\mathfrak{E}(m)^{\dagger}=\mathfrak{E}(m)$ and $\mathfrak{E}(m) \circ \mathfrak{E}(m)=\mathfrak{E}(m)$. The endomap $\mathfrak{E}(m): X \rightarrow X$ associated with a kernel/predicate $m$ on $X$ maps everything in $X$ that is in $m$ to itself, and what is perpendicular to $m$ to 0 , as expressed by the equations $\mathfrak{E}(m) \circ m=m$ and $\mathfrak{E}(m) \circ m^{\perp}=0$. Of interest is the following result. It makes the dynamical aspects of quantum logic described in [8] explicit.

Proposition 9 For kernels $m: M \longmapsto X, n: N \longmapsto X$ the pullback $\mathfrak{E}(m)^{-1}(n)$ is the Sasaki hook, written here as つ:

$$
m \supset n \stackrel{\text { def }}{=} \mathfrak{E}(m)^{-1}(n)=m^{\perp} \vee(m \wedge n) .
$$

The associated left adjoint $\exists_{\mathfrak{E}(m)} \dashv \mathfrak{E}(m)^{-1}$ yields the "and then" operator:

$$
k \& m \stackrel{\text { def }}{=} \exists_{\mathfrak{E}(m)}(k)=m \wedge\left(m^{\perp} \vee k\right),
$$

so that the "Sasaki adjunction" (see [12]) holds by construction:

$$
k \& m \leq n \Longleftrightarrow k \leq m \supset n .
$$

Quantum logic based on this "and-then" \& connective is developed in [30], see also [37, 38]. This \& connective is in general non-commutative and non-associative. ${ }^{1}$ Some basic properties are: $m \& m=m, 1 \& m=m \& 1=m, 0 \& m=m \& 0=0$, and both $k \& m \leq n, k^{\perp} \& m \leq n$ imply $m \leq n$ (which easily follows from the Sasaki adjunction).

\footnotetext{
${ }^{1}$ The "and-then" connective \& should not be confused with the multiplication of a quantale [39], since the latter is always associative.
} 
Proof Consider the following pullbacks.
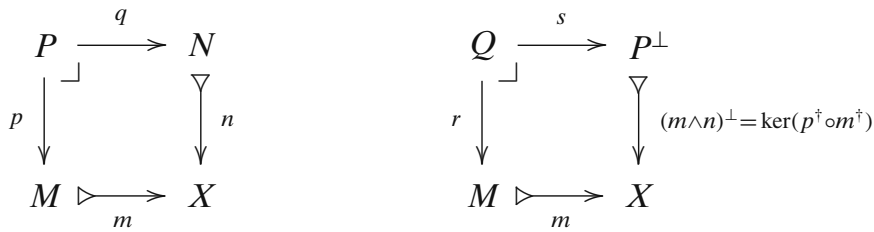

Then:

$$
\begin{aligned}
m^{\perp} \vee(m \wedge n)= & \left(m \wedge(m \wedge n)^{\perp}\right)^{\perp} \\
= & \operatorname{ker}\left(\left(m \wedge(m \wedge n)^{\perp}\right)^{\dagger}\right) \\
= & \operatorname{ker}\left(r^{\dagger} \circ m^{\dagger}\right) \\
= & \operatorname{ker}\left(\operatorname{ker}\left(\operatorname{coker}\left((m \wedge n)^{\perp}\right) \circ m\right)^{\dagger} \circ m^{\dagger}\right) \\
& \quad \text { by definition of } r \text { as pullback, see Lemma } 2 \\
= & \operatorname{ker}\left(\operatorname{ker}\left(\operatorname{coker}\left(\operatorname{ker}\left(p^{\dagger} \circ m^{\dagger}\right)\right) \circ m\right)^{\dagger} \circ m^{\dagger}\right) \\
= & \operatorname{ker}\left(\operatorname{ker}\left(p^{\dagger} \circ m^{\dagger} \circ m\right)^{\dagger} \circ m^{\dagger}\right) \\
& \quad \operatorname{because} p^{\dagger} \circ m^{\dagger} \text { is a cokernel, see Lemma 3 } \\
= & \operatorname{ker}\left(\operatorname{coker}(p) \circ m^{\dagger}\right) \\
= & \left(m^{\dagger}\right)^{-1}(p) \\
= & \left(m^{\dagger}\right)^{-1}\left(m^{-1}(n)\right) \\
= & \mathfrak{E}(m)^{-1}(n) .
\end{aligned}
$$

As we have seen, substitution functors $f^{-1}$ in dagger kernel categories have left adjoints $\exists_{f}$. It is natural to ask if they also have right adjoints $\forall_{f}$. The next result says that existence of such adjoints $\forall_{f}$ makes the logic Boolean.

Proposition 10 Suppose there are right adjoints $\forall_{f}$ to $f^{-1}: \operatorname{KSub}(Y) \rightarrow \operatorname{KSub}(X)$ for each $f: X \rightarrow Y$ in a dagger kernel category. Then each $\mathrm{KSub}(X)$ is a Boolean algebra.

Proof [25, Lemma A1.4.13] For $k, l \in \operatorname{KSub}(X)$, define implication $(k \Rightarrow l)=$ $\forall_{k}\left(k^{-1}(l)\right) \in \operatorname{KSub}(X)$. Then for any $m \in \operatorname{KSub}(X)$ :

$$
\begin{aligned}
m \leq \forall_{k}\left(k^{-1}(l)\right)=(k \Rightarrow l) & \Longleftrightarrow k^{-1}(m) \leq k^{-1}(l) \\
& \Longleftrightarrow m \wedge k=k \circ k^{-1}(m) \leq l,
\end{aligned}
$$

where the last equivalence holds because $k \circ-$ is left adjoint to $k^{-1}$, since $k$ is a kernel. Hence $\operatorname{KSub}(X)$ is a Heyting algebra, and therefore distributive. By Proposition 1 we know that it is also orthomodular. Hence each $\operatorname{KSub}(X)$ is a Boolean algebra. 
These universal quantifiers $\forall_{f}$ do not exist in general because not all kernel posets $\mathrm{KSub}(X)$ are Boolean algebras. For a concrete non-example, consider the lattice $\mathrm{KSub}\left(\mathbb{C}^{2}\right)$ in the category Hilb-where $\mathbb{C}$ denotes the complex numbers. Consider the kernel subobjects represented by

$$
\kappa_{1}: \mathbb{C} \rightarrow \mathbb{C}^{2}, \quad \kappa_{2}=\left(\kappa_{1}\right)^{\perp}: \mathbb{C} \rightarrow \mathbb{C}^{2}, \quad \Delta=\langle\text { id }, \text { id }\rangle: \mathbb{C} \rightarrow \mathbb{C}^{2} .
$$

Since we can write each $(z, w) \in \mathbb{C}^{2}$ as $(z, w)=\Delta(z, z)+\kappa_{2}(w-z)$ we get $\Delta \vee \kappa_{2}=$ 1 in $\operatorname{KSub}\left(\mathbb{C}^{2}\right)$. This yields a counterexample to distributivity:

$$
\kappa_{1} \wedge\left(\Delta \vee \kappa_{2}\right)=\kappa_{1} \wedge 1=\kappa_{1} \neq 0=0 \vee 0=\left(\kappa_{1} \wedge \Delta\right) \vee\left(\kappa_{1} \wedge \kappa_{2}\right)
$$

We now turn to a more systematic study of Booleanness. As we have seen, the categories Rel, PInj and $\widehat{B}$ (for a Boolean algebra $B$ ) are Boolean, but Hilb and PHilb are not. The following justifies the name "Boolean".

Theorem 1 A dagger kernel category is Boolean if and only if each orthomodular lattice $\mathrm{KSub}(X)$ is a Boolean algebra.

Proof We already know that each poset $\operatorname{KSub}(X)$ is an orthomodular lattice, with bottom 0 , top 1 , orthocomplement $(-)^{\perp}$ (by Lemma 1), intersections $\wedge$ (by Lemma 3), and joins $m \vee n=\left(m^{\perp} \wedge n^{\perp}\right)^{\perp}$. What is missing is distributivity $m \wedge(n \vee$ $k)=(m \vee n) \wedge(m \vee k)$. We show that the latter is equivalent to the Booleanness requirement $m \wedge n=0 \Rightarrow m \perp n$. Recall: $m \perp n$ iff $n^{\dagger} \circ m=0$ iff $m \leq n^{\perp}=\operatorname{ker}\left(n^{\dagger}\right)$.

First, assume Booleanness. In any lattice one has $m \wedge(n \vee k) \geq(m \wedge n) \vee(m \wedge k)$. For the other inequality, notice that

$$
\left(m \wedge(m \wedge n)^{\perp}\right) \wedge n=(m \wedge n) \wedge(m \wedge n)^{\perp}=0 .
$$

Hence $m \wedge(m \wedge n)^{\perp} \leq n^{\perp}$. Similarly, $m \wedge(m \wedge k)^{\perp} \leq k^{\perp}$. So

$$
m \wedge(m \wedge n)^{\perp} \wedge(m \wedge k)^{\perp} \leq n^{\perp} \wedge k^{\perp}=(n \vee k)^{\perp},
$$

and therefore

$$
m \wedge(m \wedge n)^{\perp} \wedge(m \wedge k)^{\perp} \wedge(n \vee k)=0
$$

But then we are done by using Booleanness again:

$$
m \wedge(n \vee k) \leq\left((m \wedge n)^{\perp} \wedge(m \wedge k)^{\perp}\right)^{\perp}=(m \wedge n) \vee(m \wedge k)
$$

The other direction is easier: if $m \wedge n=0$, then

$$
\begin{aligned}
m=m \wedge 1 & =m \wedge\left(n \vee n^{\perp}\right) \\
& =(m \wedge n) \vee\left(m \wedge n^{\perp}\right) \quad \text { by distributivity } \\
& =0 \vee\left(m \wedge n^{\perp}\right)=m \wedge n^{\perp},
\end{aligned}
$$

whence $m \leq n^{\perp}$. 
The Booleanness property can be strengthened in the following way.

Proposition 11 The Booleanness requirement $m \wedge n=0 \Rightarrow m \leq n^{\perp}$, for all kernels $m, n$, is equivalent to the following: for each pullback of kernels:

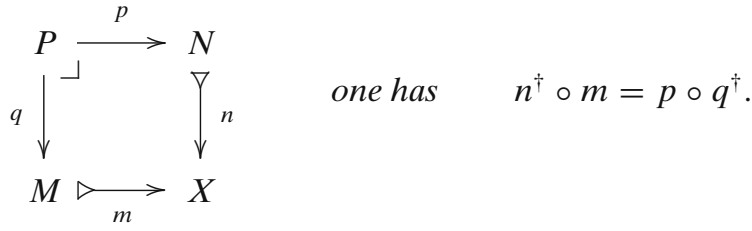

Proof It is easy to see that the definition of Booleanness is the special case $P=0$. For the converse, we put another pullback on top of the one in the statement:

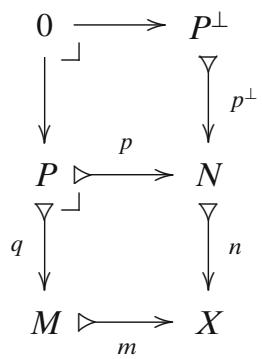

We use that $p, q$ are kernels by Lemma 2. We see $m \wedge\left(n \circ p^{\perp}\right)=0$, so by Booleanness we obtain:

$$
\begin{aligned}
m \leq\left(n \circ p^{\perp}\right)^{\perp} & =\operatorname{ker}\left(\left(n \circ \operatorname{ker}\left(p^{\dagger}\right)\right)^{\dagger}\right) \\
& =\operatorname{ker}\left(\operatorname{coker}(p) \circ n^{\dagger}\right) \\
& =\left(n^{\dagger}\right)^{-1}(p),
\end{aligned}
$$

where the pullback is as described in Lemma 2. Hence there is a map $\varphi: M \rightarrow$ $P$ with $p \circ \varphi=n^{\dagger} \circ m$. This means that $\varphi=p^{\dagger} \circ p \circ \varphi=p^{\dagger} \circ n^{\dagger} \circ m=(n \circ p)^{\dagger} \circ$ $m=(m \circ q)^{\dagger} \circ m=q^{\dagger} \circ m^{\dagger} \circ m=q^{\dagger}$. Hence we have obtained $p \circ q^{\dagger}=n^{\dagger} \circ m$, as required.

Definition 4 Let $\mathbf{D}$ be a Boolean dagger kernel category. We write $\mathbf{D}_{k c k}$ for

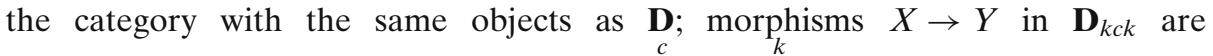
cokernel-kernel pairs $(c, k)$ of the form $X \stackrel{{ }^{c}}{\longrightarrow \bullet \triangleright}{ }^{k} \longrightarrow Y$. The identity $X \rightarrow X$ is 
$X \stackrel{\text { id }}{\longrightarrow} X \stackrel{\text { id }}{\longrightarrow} X$, and composition of $X \stackrel{{ }^{c}}{\longrightarrow} M \bowtie \stackrel{k}{\longrightarrow} Y$ and $Y \stackrel{d}{\longrightarrow} \gg N \stackrel{l}{\longrightarrow} Z$

is the pair $\left(q^{\dagger} \circ c, l \circ p\right)$ obtained via the pullback:

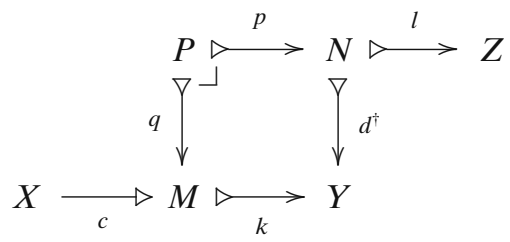

To be precise, we identity $(c, k)$ with $\left(\varphi \circ c, k \circ \varphi^{-1}\right)$, for isomorphisms $\varphi$.

The reader may have noticed that this construction generalises the definition of PInj. Indeed, now we can say PInj $=\mathbf{R e l}_{k c k}$.

Theorem 2 The category $\mathbf{D}_{k c k}$ as described in Definition 4 is again a Boolean dagger kernel category, with a functor $D: \mathbf{D}_{k c k} \rightarrow \mathbf{D}$ that is a morphism of DagKerCat, and a change-of-base situation (pullback):

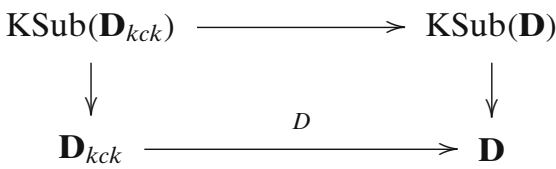

Moreover, in $\mathbf{D}_{\text {kck }}$ one has:

$$
\text { kernel }=\text { dagger mono }=\text { mono }=\text { zero-mono, }
$$

and $\mathbf{D}_{k c k}$ is universal among such categories.

Proof The obvious definition $(c, k)^{\dagger}=\left(k^{\dagger}, c^{\dagger}\right)$ yields an involution on $\mathbf{D}_{k c k}$. The zero object $0 \in \mathbf{D}$ is also a zero object $0 \in \mathbf{D}_{k c k}$ with zero map $X \longrightarrow 0 \triangleright Y$ consisting of a cokernel-kernel pair. A map $(c, k)$ is a dagger mono if and only if $(c, k)^{\dagger} \circ(c, k)=$ $\left(k^{\dagger}, k\right)$ is the identity; this means that $k=\mathrm{id}$.

The kernel of a map $\left.(d, l)=\left(Y \stackrel{d}{\longrightarrow} d_{\text {id }}^{\perp}\right)^{\perp} \stackrel{l}{\longrightarrow} Z\right)$ in $\mathbf{D}_{k c k}$ is $\operatorname{ker}(d, l)=$ $\left(N^{\perp} \longrightarrow N^{\perp} \longmapsto Y\right.$ ), so that $\operatorname{ker}(d, l)$ is a dagger mono and $(d, l) \circ \operatorname{ker}(d, l)=0$. If also $(d, l) \circ(c, k)=0$, then $k \wedge d^{\dagger}=0$ so that by Booleanness, $k \leq\left(d^{\dagger}\right)^{\perp}$, say via $\varphi: M \rightarrow N^{\perp}$ with $\left(d^{\dagger}\right)^{\perp} \circ \varphi=k$. Then we obtain a mediating map

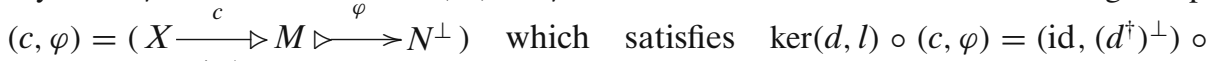
$(c, \varphi)=\left(c,\left(d^{\dagger}\right)^{\perp} \circ \varphi\right)=(c, k)$. It is not hard to see that maps of the form (id, $m$ ) in $\mathbf{D}_{k c k}$ are kernels, namely of the cokernel $\left(m^{\perp}, \mathrm{id}\right)$.

The intersection of two kernels (id, $m)=(M=M \stackrel{m}{\longmapsto} X)$ and (id, $n)=$ $\left(N=N \triangleright^{n}>X\right)$ in $\mathbf{D}_{k c k}$ is the intersection $m \wedge n: P \longmapsto X$ in $\mathbf{D}$, with projections $(P=P \triangleright M$ ) and ( $P=P \triangleright>N$ ). Hence if the intersection of (id, $m$ ) and (id, $n)$ in $\mathbf{D}_{k c k}$ is 0 , then so is the intersection of $m$ and $n$ in $\mathbf{D}$, which yields 
$n^{\dagger} \circ m=0$. But then in $\mathbf{D}_{k c k}$, (id, $\left.n\right)^{\dagger} \circ(\mathrm{id}, m)=\left(n^{\dagger}, \mathrm{id}\right) \circ(\mathrm{id}, m)=0$. Hence $\mathbf{D}_{k c k}$ is also Boolean.

Finally, there is a functor $\mathbf{D}_{k c k} \rightarrow \mathbf{D}$ by $X \mapsto X$ and $(c, k) \mapsto k \circ c$. Composition is preserved by Proposition 11, since for maps as in Definition 4 ,

$$
(d, l) \circ(c, k)=\left(q^{\dagger} \circ c, l \circ p\right) \longmapsto l \circ p \circ q^{\dagger} \circ c=(l \circ d) \circ(k \circ c) .
$$

We have already seen that $\mathrm{KSub}(X)$ in $\mathbf{D}_{k c k}$ is isomorphic to $\mathrm{KSub}(X)$ in $\mathbf{D}$. This yields the change-of-base situation.

We have already seen that kernels and dagger monos coincide. We now show that they also coincide with zero-monos. So let $(d, l): Y \rightarrow Z$ be a zero-mono. This means that $(d, l) \circ(c, k)=0 \Rightarrow(c, k)=0$, for each map $(c, k)$. Using diagram $(8)$, this means: $d^{\dagger} \wedge k=0 \Rightarrow k=0$. By Booleanness, the antecedent $d^{\dagger} \wedge k=0$ is equivalent to $k \leq\left(d^{\dagger}\right)^{\perp}=\operatorname{ker}(d)$, which means $d \circ k=0$. Hence we see that $d$ is zero-monic in $\mathbf{D}$, and thus an isomorphism (because it is already a cokernel).

Finally, let $\mathbf{E}$ be a Boolean dagger kernel category in which zero-monos are kernels, with a functor $F: \mathbf{E} \rightarrow \mathbf{D}$ in DagKerCat. Every morphism $f$ in $\mathbf{E}$ factors as $f=i_{f} \circ e_{f}$ for a kernel $i_{f}$ and a cokernel $e_{f}$. Hence $G: \mathbf{E} \rightarrow \mathbf{D}_{k c k}$ defined by $G(X)=F(X)$ and $G(f)=\left(e_{f}, i_{f}\right)$ is the unique functor satisfying $F=D \circ G$.

\section{Ordering Homsets}

This section shows that homsets in dagger kernel categories automatically carry a partial order. However, this does not make the categories order enriched, because the order is not preserved by all morphisms.

Definition 5 Let $f, g: X \rightarrow Y$ be parallel morphisms in a dagger kernel category. After factorising them as $f=i_{f} \circ m_{f} \circ\left(i_{f^{\dagger}}\right)^{\dagger}$ and $g=i_{g} \circ m_{g} \circ\left(i_{g^{\dagger}}\right)^{\dagger}$ like in (7) we can define $f \leq g$ if and only if there are (necessarily unique, dagger monic) $\varphi: \operatorname{Im}(f) \rightarrow \operatorname{Im}(g)$ and $\psi: \operatorname{Im}\left(f^{\dagger}\right) \rightarrow \operatorname{Im}\left(g^{\dagger}\right)$, so that in the diagram

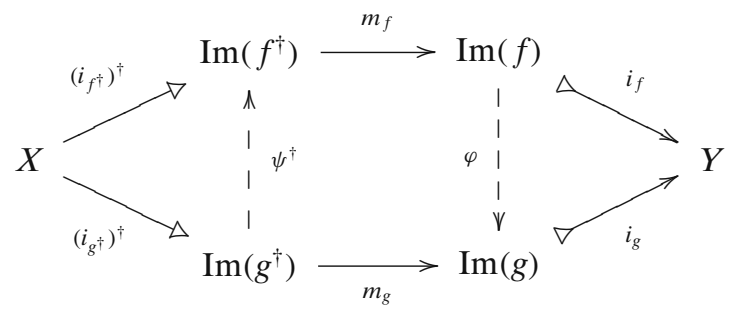

one has

$$
\psi^{\dagger} \circ\left(i_{g^{\dagger}}\right)^{\dagger}=\left(i_{f^{\dagger}}\right)^{\dagger} \quad \varphi \circ m_{f}=m_{g} \circ \psi \quad \varphi^{\dagger} \circ m_{g}=m_{f} \circ \psi^{\dagger} \quad i_{g} \circ \varphi=i_{f} .
$$

Lemma 9 The relation $\leq$ is a partial order on each homset of a dagger kernel category, with the zero morphism as least element.

Proof Reflexivity is easily established by taking $\varphi=\mathrm{id}$ and $\psi=\mathrm{id}$ in (9). For transitivity, suppose that $f \leq g$ via $\varphi$ and $\psi$, and that $g \leq h$ via $\alpha$ and $\beta$. Then the 
four conditions in the previous definition are fulfilled by $\alpha \circ \varphi$ and $\psi \circ \beta$, so that $f \leq h$. Finally, for anti-symmetry, suppose that $f \leq g$ via $\varphi$ and $\psi$, and that $g \leq f$ via $\alpha$ and $\beta$. Then $i_{f} \circ \alpha \circ \varphi=i_{g} \circ \varphi=i_{f}$, so that $\alpha \circ \varphi=$ id. Similarly, $\beta \circ \psi=\mathrm{id}$. By Lemma 1, $\alpha$ is a dagger mono so that $\alpha^{\dagger}=\alpha^{\dagger} \circ \alpha \circ \varphi=\varphi$. Similarly, $\beta^{\dagger}=\psi$, and thus:

$$
\begin{aligned}
f=i_{f} \circ m_{f} \circ\left(i_{f^{\dagger}}\right)^{\dagger}=i_{f} \circ \alpha \circ \varphi \circ m_{f} \circ\left(i_{f^{\dagger}}\right)^{\dagger} & =i_{g} \circ m_{g} \circ \psi \circ\left(i_{f^{\dagger}}\right)^{\dagger} \\
& =i_{g} \circ m_{g} \circ \beta^{\dagger} \circ\left(i_{f^{\dagger}}\right)^{\dagger} \\
& =i_{g} \circ m_{g} \circ\left(i_{g^{\dagger}}\right)^{\dagger} \\
& =g .
\end{aligned}
$$

Finally, for any $f$ we have $0 \leq f$ by taking $\varphi=\psi=0$ in (9).

Lemma 10 If $f \leq g$, then:

1. $(k \circ f) \leq(k \circ g)$ for a kernel $k$;

2. $(f \circ c) \leq(g \circ c)$ for a cokernel $c$;

3. $f^{\dagger} \leq g^{\dagger}$.

Proof The first two points are obvious. The third one then follows because $\left(m_{f}\right)^{\dagger}=$ $m_{f^{\dagger}}$ as shown in Section 5 .

Example 4 We describe the situation in PInj, Rel and Hilb, using the factorisations from Example 3.

Two parallel maps $f=\left(X \stackrel{f_{1}}{\longleftarrow} F \stackrel{f_{2}}{\longmapsto} Y\right)$ and $g=\left(X \stackrel{g_{1}}{\longleftarrow} G \stackrel{g_{2}}{\longmapsto} Y\right)$ in PInj satisfy $f \leq g$ if and only if there are $\varphi, \psi: F \rightarrow G$ in:

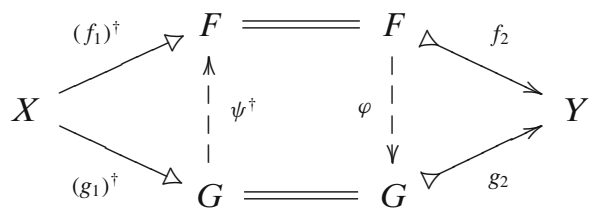

This means $\varphi=\psi$ and $g_{i} \circ \varphi=f_{i}$, for $i=1,2$, so that we obtain the usual order (of one partial injection extending another).

Next, $R \leq S$ for $R=\left(X \stackrel{r_{1}}{\leftarrow} R \stackrel{r_{2}}{\rightarrow} Y\right)$ and $S=\left(X \stackrel{s_{1}}{\leftarrow} S \stackrel{s_{2}}{\rightarrow} Y\right)$ in Rel means:

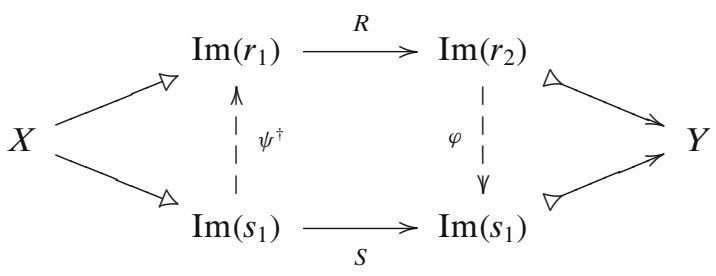


Commutation of the triangles means $\operatorname{Im}\left(r_{1}\right) \subseteq \operatorname{Im}\left(s_{1}\right)$ and $\operatorname{Im}\left(r_{2}\right) \subseteq \operatorname{Im}\left(s_{2}\right)$. The equations for the square in the middle say that:

$$
R(x, y) \Leftrightarrow S(x, y) \text { for all }\left\{\begin{array}{l}
(x, y) \in \operatorname{Im}\left(r_{1}\right) \times \operatorname{Im}\left(s_{2}\right) \\
(x, y) \in \operatorname{Im}\left(r_{2}\right) \times \operatorname{Im}\left(s_{1}\right)
\end{array}\right.
$$

This means $R \subseteq S$, as one would expect.

The order on the homsets of the category Hilb can be characterized as follows [20, Example 5.1.10]: $f \leq g$ for $f, g: X \rightarrow Y$ if and only if $g=f+f^{\prime}$ for some $f^{\prime}: X \rightarrow Y$ with $\operatorname{Im}(f)$ and $\operatorname{Im}\left(f^{\dagger}\right)$ orthogonal to $\operatorname{Im}\left(f^{\prime}\right)$ and $\operatorname{Im}\left(\left(f^{\prime}\right)^{\dagger}\right)$, respectively. To see this, suppose that $g=f+f^{\prime}$ as above. Then $\operatorname{Im}(g)$ is the direct sum of $\operatorname{Im}(f)$ and $\operatorname{Im}\left(f^{\prime}\right)$, and likewise $\operatorname{Im}\left(g^{\dagger}\right)=\operatorname{Im}\left(f^{\dagger}\right) \oplus \operatorname{Im}\left(\left(f^{\prime}\right)^{\dagger}\right.$. Moreover, $m_{g}$ is the direct sum of $m_{f}$ and $m_{f^{\prime}}$. Therefore, taking $\psi=\varphi=\kappa_{1}$ makes diagram (9) commute, so that $f \leq g$. Conversely, suppose that $f \leq g$, so that diagram (9) commutes. Then the cotuple $\left[\varphi, \varphi^{\perp}\right]: \operatorname{Im}(f) \oplus \operatorname{Im}(f)^{\perp} \rightarrow \operatorname{Im}(g)$ is an isomorphism, and so is the cotuple $\left[\psi, \psi^{\perp}\right]$. Since $\varphi^{\dagger} \circ m_{g}=m_{f} \circ \psi^{\dagger}$, there is a morphism $n$ making the following diagram commute:

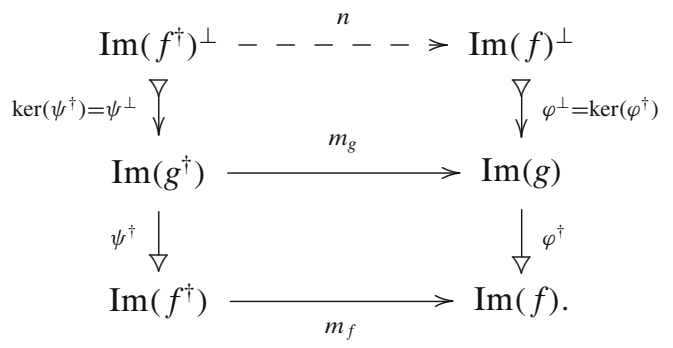

Now, taking

$$
f^{\prime}=\left(X \stackrel{\left(i_{g^{\dagger}}\right)^{\dagger}}{\longrightarrow} \operatorname{Im}\left(g^{\dagger}\right) \stackrel{\left(\psi^{\perp}\right)^{\dagger}}{\longrightarrow} \operatorname{Im}\left(f^{\dagger}\right)^{\perp} \stackrel{n}{\longrightarrow} \operatorname{Im}(f)^{\perp} \stackrel{\varphi^{\perp}}{\longrightarrow} \operatorname{Im}(g) \stackrel{i_{g}}{\longrightarrow} Y\right)
$$

fulfills $g=f+f^{\prime}$, and $\operatorname{Im}(f)$ and $\operatorname{Im}\left(f^{\dagger}\right)$ are orthogonal to $\operatorname{Im}\left(f^{\prime}\right)$ and $\operatorname{Im}\left(\left(f^{\prime}\right)^{\dagger}\right)$, respectively.

In Hilbert spaces there is a standard correspondence between self-adjoint idempotents and closed subsets. Recall that an endomap $p: X \rightarrow X$ is self-adjoint if $p^{\dagger}=p$ and idempotent if $p \circ p=p$. In the current, more general, setting this works as follows, using the order on homsets. 
Proposition 12 The "effect" mapping $m \mapsto \mathfrak{E}(m) \stackrel{\text { def }}{=} m \circ m^{\dagger}$ from Section 6 yields an order isomorphism:

$$
\begin{aligned}
\operatorname{KSub}(X) & \cong\left\{p: X \rightarrow X \mid p^{\dagger}=p \leq \mathrm{id}\right\} \\
& \cong\left\{p: X \rightarrow X \mid p^{\dagger}=p \circ p=p \leq \mathrm{id}\right\} \\
& \stackrel{(*)}{\cong}\left\{p: X \rightarrow X \mid p^{\dagger}=p \circ p=p\right\},
\end{aligned}
$$

where the marked isomorphism holds if zero-epis are epis (like in Hilb).

Proof Clearly, $\mathfrak{E}(m)=m \circ m^{\dagger}$ is a self-adjoint idempotent. It satisfies $\mathfrak{E}(m) \leq$ id via:

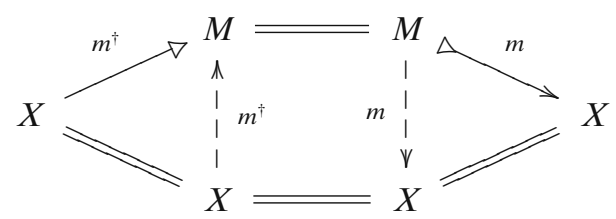

where the kernel $m: M \longmapsto X$ is a dagger mono so that $\operatorname{Im}(\mathfrak{E}(m))=M$.

This mapping $\mathfrak{E}(-): \operatorname{KSub}(X) \rightarrow\left\{p \mid p^{\dagger}=p \leq \mathrm{id}\right\}$ is surjective: if $p: X \rightarrow X$ is a self-adjoint with $p \leq$ id then we first note that the factorisation from (7) yields $p=i_{p} \circ m_{p} \circ\left(i_{p}\right)^{\dagger}$. By Definition 5 there are $\varphi, \psi: \operatorname{Im}(p) \rightarrow X$ with $\psi^{\dagger}=\left(i_{p}\right)^{\dagger}$, $\varphi \circ m_{p}=\psi, \varphi^{\dagger}=m_{p} \circ \psi^{\dagger}$ and $\varphi=i_{p}$. This yields $\psi=i_{p}$ and $m_{p}=$ id. Hence $p=$ $i_{p} \circ\left(i_{p}\right)^{\dagger}=\mathfrak{E}\left(i_{p}\right)$, so that $p$ is automatically idempotent. This establishes the second isomorphism.

The mapping $\mathfrak{E}(-)$ preserves and reflects the order. If $m \leq n$ in $\mathrm{KSub}(X)$, say via $\varphi: M \rightarrow N$ with $n \circ \varphi=m$, then $\mathfrak{E}(m) \leq \mathfrak{E}(n)$ via:

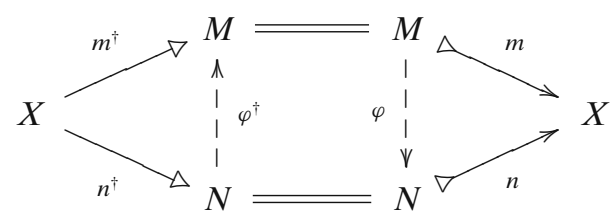

Conversely, if $\mathfrak{E}(m) \leq \mathfrak{E}(n)$, say via $\varphi: M \rightarrow N$ and $\psi: M \rightarrow N$, then $n \circ \varphi=m$ so that $m \leq n$ in $\operatorname{KSub}(X)$.

Finally, if zero-epis are epis, we write for a self-adjoint idempotent $p$,

$$
i_{p} \circ e_{p}=p=p \circ p=p^{\dagger} \circ p=\left(e_{p}\right)^{\dagger} \circ\left(i_{p}\right)^{\dagger} \circ i_{p} \circ e_{p}=\left(e_{p}\right)^{\dagger} \circ e_{p},
$$

and obtain $i_{p}=\left(e_{p}\right)^{\dagger}$. Hence $p=\mathfrak{E}\left(i_{p}\right)$ and thus $p \leq \mathrm{id}$.

\footnotetext{
${ }^{2}$ The name "effect" was chosen because of connections to effect algebras [11]. For example, in the so-called standard effect algebra of a Hilbert space [13], an effect corresponds a positive operator beneath the identity.
} 


\section{Completeness and Atomicity of Kernel Posets}

In traditional quantum logic, orthomodular lattices are usually considered with additional properties, such as completeness and atomicity [34]. This section considers how these requirements on the lattices $\operatorname{KSub}(X)$ translate to categorical properties. For convenience, let us recall the following standard order-theoretical definitions.completeness

Definition 6 For elements $x, y$ of a poset, we say that $y$ covers $x$ when $x<y$ and $x \leq z<y$ implies $z=x$ (where $z<y$ if and only if $z \leq y$ and $z \neq y$ ). An element $a$ of a poset with least element 0 is called an atom when it covers 0 . Equivalently, an atom cannot be expressed as a join of strictly smaller elements. Consequently, 0 is not an atom. A poset is called atomic if for any $x \neq 0$ in it there exists an atom $a$ with $a \leq x$. Finally, a lattice is atomistic when every element is a join of atoms [10].

Proposition 13 For an arbitrary object I in a dagger kernel category, the following are equivalent:

1. $\operatorname{id}_{I}=1$ is an atom in $\mathrm{KSub}(I)$;

2. $\operatorname{KSub}(I)=\{0,1\}$;

3. each nonzero kernel $x: I \longmapsto X$ is an atom in $\operatorname{KSub}(X)$.

Proof For the implication (1) $\Rightarrow(2)$, let $m$ be a kernel into $I$. Because $m \leq \mathrm{id}_{I}$ and the latter is an atom, we have that $m=0$ or $m$ is isomorphism. Thus $\operatorname{KSub}(I)=\{0,1\}$.

To prove $(2) \Rightarrow(3)$, suppose that $m \leq x$ for kernels $m: M \longmapsto X$ and $x: I \longmapsto X$. Say $m=x \circ \varphi$ for $\varphi: M \longmapsto I$. Then $\varphi$ is a kernel by Lemma 1 . Since $\operatorname{KSub}(I)=\{0,1\}$, either $\varphi$ is zero or $\varphi$ is isomorphism. Hence either $m=0$ or $m=x$ as subobjects. So $x$ is an atom. Finally, (3) $\Rightarrow$ (1) is trivial.

Definition 7 If $I$ satisfies the conditions of the previous lemma, we call it a KSubsimple object. (Any simple object in the usual sense of category theory is KSubsimple.)

Similarly, let us call $I$ a KSub-generator if $f=g: X \rightarrow Y$ whenever $f \circ x=g \circ x$ for all kernels $x: I \longmapsto X$. (Any KSub-generator is a generator in the usual sense of category theory.)

Example 5 The objects $1 \in \mathbf{P I n j}, 1 \in \mathbf{R e l}, \mathbb{C} \in \mathbf{H i l b}$ and $\mathbb{C} \in \mathbf{P H i l b}$ are KSub-simple KSub-generators.

The two-element orthomodular lattice 2 is a generator in the category OMLatGal from [22], because maps $2 \rightarrow X$ correspond to elements in $X$. But 2 is not a KSubgenerator: these maps $2 \rightarrow X$ are not kernels.

Because $1 \in$ Rel is a KSub-simple KSub-generator, one might expect a connection between Definition 7 and the "kernel opclassifiers" discussed at the end of Section 4. There is, however, no apparent such connection. For example, the object 1 in the category PInj is a KSub-simple KSub-generator, but not a "kernel opclassifier".

Lemma 11 Suppose that a dagger kernel category D has a KSub-simple KSubgenerator I. Then beneath any nonzero element of $\mathrm{KSub}(X)$ lies a nonzero element of 
the form $x: I \longmapsto X$. Hence $\mathrm{KSub}(X)$ is atomic, and its atoms are the nonzero kernels $x: I \longmapsto X$.

Proof Suppose $m: M \longmapsto X$ is a nonzero kernel. Since $I$ is a KSub-generator, there must be a kernel $x: I \longmapsto M$ with $m \circ x \neq 0$. By Proposition 13 this $m \circ x$ is an atom. It satisfies $m \circ x \leq m$, so we are done.

Corollary 2 If a dagger kernel category has a KSub-simple KSub-generator I, then $\operatorname{KSub}(X)$ is atomistic for any object $X$.

Proof Any atomic orthomodular lattice is atomistic [26].

The categorical requirement of a simple generator is quite natural in this setting, as it is also used to prove that a certain class of dagger kernel categories embeds into Hilb [19].

We now turn to completeness, by showing that the existence of directed colimits ensures that kernel subobject lattices are complete. This, too, is a natural categorical requirement in the context of infinite-dimensionality [17]. Recall that a directed colimit is a colimit of a directed poset, considered as a diagram. The following result can be obtained abstractly in two steps: directed colimits in $\mathbf{D}$ yield direct colimits in slice categories $\mathbf{D} / X$, see [5, Vol. 2, Prop. 2.16.3]. The reflection $\operatorname{KSub}(X) \hookrightarrow \mathbf{D} / X$ induced by factorisation transfers these directed colimits to $\operatorname{KSub}(X)$. However, in the proof below we give a concrete construction.

Proposition 14 If a dagger kernel category $\mathbf{D}$ has directed colimits, then $\operatorname{KSub}(X)$ is a complete lattice for every $X \in \mathbf{D}$.

Proof A lattice is complete if it has directed joins (see [24, Lemma I.4.1], or [27, Lemma 2.12]), so we shall prove that $\operatorname{KSub}(X)$ has such directed joins. Let $\left(m_{i}: M_{i} \longmapsto X\right)_{i \in I}$ be a directed collection in $\operatorname{KSub}(X)$. For $i \leq j$ we have $m_{i} \leq m_{j}$ and thus $m_{j} \circ m_{j}^{\dagger} \circ m_{i}=m_{i}$.

Let $M$ be the colimit in $\mathbf{D}$ of the domains $M_{i}$, say with coprojections $c_{i}: M_{i} \rightarrow$ $M$. The $\left(m_{i}: M_{i} \longmapsto X\right)_{i \in I}$ form a cocone by assumption, so there is a unique map $m: M \rightarrow X$ with $m \circ c_{i}=m_{i}$. The kernel/zero-epi factorisation (6) yields:

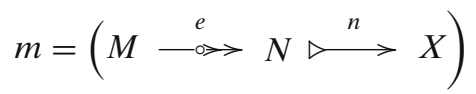

We claim that $n$ is the join in $\operatorname{KSub}(X)$ of the $m_{i}$.

- $\quad m_{i} \leq n$ via $e \circ c_{i}: M_{i} \rightarrow N$ satisfying $n \circ\left(e \circ c_{i}\right)=m \circ c_{i}=m_{i}$.

- If $m_{i} \leq k$, then $k \circ k^{\dagger} \circ m_{i}=m_{i}$. Also, the maps $k_{i}=k^{\dagger} \circ m_{i}: M_{i} \rightarrow K$ form a cocone in $\mathbf{D}$ because the $m_{i}$ are directed and $k$ is monic: if $i \leq j$, then,

$$
k \circ k_{j} \circ m_{j}^{\dagger} \circ m_{i}=k \circ k^{\dagger} \circ m_{j} \circ m_{j}^{\dagger} \circ m_{i}=k \circ k^{\dagger} \circ m_{i}=k \circ k_{i} .
$$

As a result there is a unique $\ell: M \rightarrow K$ with $\ell \circ c_{i}=k_{i}$. Then $k \circ \ell=m$ by uniqueness since:

$$
k \circ \ell \circ c_{i}=k \circ k_{i}=k \circ k^{\dagger} \circ m_{i}=m_{i}=m \circ c_{i} .
$$


Hence we obtain $n \leq k$ by diagonal-fill-in from Lemma 7 in:

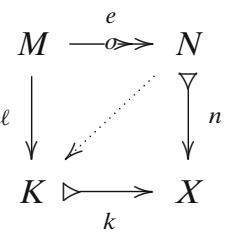

Example 6 The categories PInj, Rel, Hilb and PHilb have directed colimits, and therefore their kernel subobject lattices are complete orthomodular lattices. Since they also have appropriate generators, see Example 5, each $\operatorname{KSub}(X)$ in PInj, Rel, Hilb or PHilb is a complete atomic atomistic orthomodular lattice.

Any atom of a Boolean algebra $B$ is a KSub-simple object in the dagger kernel category $\widehat{B}$ from Proposition 6 . But $\widehat{B}$ has a KSub-generator only if $B$ is atomistic. In that case the greatest element 1 is a KSub-generator. For if $f \circ a=g \circ a$ for all $a \leq 1 \wedge x=x$ and $f, g \leq x \wedge y$, then, writing $\downarrow_{A} x=\{a \in \operatorname{Atoms}(B) \mid a \leq x\}$ we get:

$$
\begin{aligned}
f=f \wedge x=f \wedge\left(\bigvee \downarrow_{A} x\right) & =\bigvee\{f \wedge a \mid a \in \operatorname{Atoms}(B), a \leq x\} \\
& =\bigvee\{g \wedge a \mid a \in \operatorname{Atoms}(B), a \leq x\} \\
& =g \wedge\left(\bigvee \downarrow_{A} x\right)=g \wedge x=g .
\end{aligned}
$$

\section{Conclusions and Future Work}

The paper shows that a "dagger kernel category" forms a simple but powerful notion that not only captures many examples of interest in quantum logic but also provides basic structure for categorical logic. There are many avenues for extension and broadening of this work, by including more examples (e.g. effect algebras [11]) or more structure (like tensors). Also, integrating probabilistic aspects of quantum logic is a challenge.

Acknowledgements Thanks are due to an anonymous referee for pointing out the reference [9]. It already contains several early ideas that are rediscovered and elaborated in the current (categorical) setting. The category OMLatGal that plays a central role in [22] is also already in [9].

Open Access This article is distributed under the terms of the Creative Commons Attribution Noncommercial License which permits any noncommercial use, distribution, and reproduction in any medium, provided the original author(s) and source are credited.

\section{References}

1. Abramsky, S., Coecke, B.: A categorical semantics of quantum protocols. In: Logic in Computer Science, IEEE, pp. 415-425 (2004)

2. Awodey, S.: Category Theory. Oxford Logic Guides, Oxford Univ. Press, Oxford (2006)

3. Barr, M.: Algebraically compact functors. J. Pure Appl. Algebra 82, 211-231 (1992)

4. Barr, M., Wells, C.: Toposes, Triples and Theories. Springer, Berlin. Revised and corrected version available from URL: www.cwru.edu/artsci/math/wells/pub/ttt.html (1985) 
5. Borceux, F.: Handbook of Categorical Algebra. Encyclopedia of Mathematics 50, 51 and 52. Cambridge Univ. Press, Cambridge (1994)

6. Cassinelli, G., De Vito, E.P., Lahti J., Levrero, A.: The Theory of Symmetry Actions in Quantum Mechanics. Number 654 in Lecture Notes in Physics. Springer, Berlin (2004)

7. Coecke, B., Pavlović, D.: Quantum measurements without sums. In: Chen, G., Kauffman, L., Lamonaco, S. (eds.) Mathematics of Quantum Computing and Technology. Taylor and Francis, Philadelphia. See also: arXiv:/quant-ph/0608035 (2006)

8. Coecke, B., Smets, S.: The Sasaki hook is not a [static] implicative connective but induces a backward [in time] dynamic one that assigns causes. Int. J. Theor. Phys. 43(7/8), 1705-1736 (2004)

9. Crown, G.: On some orthomodular posets of vector bundles. J. Nat. Sci. Math. 15(1-2), 11-25 (1975)

10. Davey, B., Priestley, H.: Introduction to Lattices and Order. Math. Textbooks. Cambridge Univ. Press, Cambridge (1990)

11. Dvurečenskij, A., Pulmannová, S.: New Trends in Quantum Structures. Kluwer Acad., Dordrecht (2000)

12. Finch, P.D.: Quantum logic as an implication algebra. Bull. Am. Math. Soc. 2, 101-106 (1970)

13. Foulis, D.J., Greechie, R., Bennett, M.: Sums and products of interval algebras. Int. J. Theor. Phys. 33(11), 2119-2136 (1994)

14. Freyd, P.: Abelian Categories: An Introduction to the Theory of Functors. Harper and Row, New York. Available via www.tac.mta.ca/tac/reprints/articles/3/tr3.pdf (1964)

15. Haghverdi, E., Scott, P.: A categorical model for the geometry of interaction. Theor. Comp. Sci. 350, 252-274 (2006)

16. Harding, J.: Orthomodularity of decompositions in a categorical setting. Int. J. Theor. Phys. 45(6), 1117-1128 (2006)

17. Heunen, C.: Compactly accessible categories and quantum key distribution. Logical Methods Comp. Sci. 4(4) (2008). doi:10.2168/LMCS-4(4:9)2008

18. Heunen, C.: Quantifiers for quantum logic. arXiv:0811.1457 (2008)

19. Heunen, C.: An embedding theorem for Hilbert categories. Theory Appl. Categ. 22, 321-344 (2009)

20. Heunen, C.: Categorical quantum models and logics. Ph.D. thesis, Radboud Univ. Nijmegen (2010)

21. Jacobs, B.: Categorical Logic and Type Theory. North Holland, Amsterdam (1999)

22. Jacobs, B.: Orthomodular lattices, Foulis semigroups and dagger kernel categories. Logical Methods Comp. Sci. (2009). Available from http://arxiv.org/abs/0905.4090

23. Janowitz, M.F.: Quantifiers and orthomodular lattices. Pac. J. Math. 13, 1241-1249 (1963)

24. Johnstone, P.: Stone Spaces. Number 3 in Cambridge Studies in Advanced Mathematics. Cambridge Univ. Press, Cambridge (1982)

25. Johnstone, P.T.: Sketches of an Elephant: A Topos Theory Compendium. Oxford University Press, Oxford (2002)

26. Kalmbach, G.: Orthomodular Lattices. Academic, London (1983)

27. Kalmbach, G.: Measures and Hilbert Lattices. World Scientific, Singapore (1986)

28. Kock, A., Reyes, G.: Doctrines in categorical logic. In: Barwise, J. (ed.) Handbook of Mathematical Logic, pp. 283-313. North-Holland, Amsterdam (1977)

29. Lane, S.M.: Categories for the Working Mathematician. Springer, Berlin (1971)

30. Lehmann, D.: A presentation of quantum logic based on an and then connective. J. Log. Comput. 18(1), 59-76 (2008)

31. Mac Lane, S.: An algebra of additive relations. Proc. Natl. Acad. sci. 47, 1043-1051 (1961)

32. Makkai, M., Reyes, G.: First Order Categorical Logic. Number 611 in Lect. Notes Math., Springer, Berlin (1977)

33. Manes, E.: Monads, matrices and generalized dynamic algebra. In: Ehrig, H., Herrlich, H., Kreowski, H.-J., Preuß, G. (eds.) Categorical Methods in Computer Science With Aspects from Topology, number 393 in Lect. Notes Comp. Sci, pp. 66-81 (1989)

34. Piron, C.: Foundations of Quantum Physics. Number 19 in Mathematical Physics Monographs. Benjamin, New York (1976)

35. Puppe, D.: Korrespondenzen in abelschen Kategorien. Math. Ann. 148, 1-30 (1962)

36. Román, L.: A characterization of quantic quantifiers in orthomodular lattices. Theory Appl. Categ. 16, 206-217 (2006)

37. Román, L., Rumbos, B.: A characterization of nuclei in orthomodular and quantic lattices. J. Pure Appl. Algebra 73, 155-163 (1991) 
38. Román, L., Zuazua, R.: On quantic conuclei in orthomodular lattices. Theory Appl. Categ. 2(6), 62-68 (1996)

39. Rosenthal, K.: Quantales and Their Applications. Number 234 in Pitman Research Notes in Math. Longman Scientific \& Technical, Harlow (1990)

40. Selinger, P.: Dagger compact closed categories and completely positive maps (extended abstract). In: Selinger, P. (ed.) Proceedings of the 3rd International Workshop on Quantum Programming Languages (QPL 2005), number 170 in Elect. Notes in Theor. Comp. Sci., pp. 139-163. http://dx.doi.org/10.1016/j.entcs.2006.12.018 (2007)

41. Taylor, P.: Practical Foundations of Mathematics. Number 59 in Cambridge Studies in Advanced Mathematics. Cambridge Univ. Press, Cambridge (1999) 